Geometry 85 Topology

Volume 5 (2001) 761-797

Published: 25 October 2001

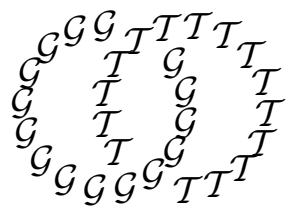

\title{
Instantons on cylindrical manifolds and stable bundles
}

\author{
BRENDAN OWENS \\ Department of Mathematics and Statistics \\ McMaster University \\ Hamilton, Ontario, Canada \\ Email: owensb@icarus.math.mcmaster.ca
}

\begin{abstract}
Let $\Sigma$ be a smooth complex curve, and let $S$ be the product ruled surface $\Sigma \times \mathbf{C P}^{\mathbf{1}}$. We prove a correspondence conjectured by Donaldson between finite energy $U(2)$-instantons over $\Sigma \times S^{1} \times \mathbf{R}$, and rank 2 holomorphic bundles over $S$ whose restrictions to $\Sigma \times\{0\}, \Sigma \times\{\infty\}$ are stable.
\end{abstract}

\section{AMS Classification numbers Primary: $53 \mathrm{C} 07$}

Secondary: 14J60, 57R58, 14J80

Keywords: Anti-self-dual connection, stable bundle, product ruled surface

Proposed: Simon Donaldson

Seconded: John Morgan, Tomasz Mrowka

(c) Geometry $8 \mathcal{G}$ Topology $\mathcal{P}$ ublications
Received: 23 February 2001

Revised: 25 October 2001 


\section{Introduction}

Let $\Sigma$ be a smooth complex curve and let $Y=\Sigma \times S^{1}$. Let $E \rightarrow \Sigma \times S^{1} \times \mathbf{R}$ be a rank 2 complex vector bundle pulled back from $\Sigma$, with $c_{1}=1$ over $\Sigma$.

Denote by $\mathcal{M}$ the moduli space of finite energy $U(2)$-instantons on $E$. There is a natural involution on $\mathcal{M}$ which we denote by $\mathcal{I}$. This is essentially given by tensoring $E$ with the flat complex line bundle pulled back from the nontrivial double cover of the $S^{1}$ factor.

The cylinder $\Sigma \times S^{1} \times \mathbf{R}$ may be compactified by adding a copy of $\Sigma$ at each end. We denote these curves by $\Sigma_{0}$ and $\Sigma_{\infty}$. The resulting compactification is the product ruled surface $S=\Sigma \times \mathbf{C P}^{\mathbf{1}}$. Let $\mathcal{Z}$ denote the space of isomorphism classes of rank 2 holomorphic bundles over $S$ with fixed degree 1 determinant over $\Sigma$ and whose restriction to $\Sigma_{0}$ and $\Sigma_{\infty}$ is stable.

The following result was conjectured by Donaldson in [5]:

Theorem 1 The space $\mathcal{M} / \mathcal{I}$ is naturally homeomorphic to $\mathcal{Z}$.

(The topologies on the spaces $\mathcal{M} / \mathcal{I}$ and $\mathcal{Z}$ are quotients of the $C^{\infty}$ topologies on compact sets, see Sections 3 and 5 for details.)

There are four main steps in the proof of Theorem 1. First we define a map

$$
\Psi: \mathcal{M} / \mathcal{I} \rightarrow \mathcal{Z}
$$

Given $[A] \in \mathcal{M}$ we obtain $\Psi([A])$ by extending the holomorphic bundle $\left(E, \bar{\partial}_{A}\right)$ over $\Sigma_{0}$ and $\Sigma_{\infty}$. The idea behind this extension is a simple one which exploits the product structure of the $3-$ manifold $\Sigma \times S^{1}$. Standard convergence results for finite energy instantons enable us to show that the restriction of $\left(E, \bar{\partial}_{A}\right)$ to every fiber $\Sigma \times(\theta, t) \subset \Sigma \times S^{1} \times \mathbf{R}$ is stable for large $|t|$. Thus the bundle on each end of the tube gives a holomorphic map from a punctured disk into the moduli space $\mathcal{N}(\Sigma)$ of stable bundles on $\Sigma$; by removable singularities we are able to extend this map over the puncture and thus also extend the bundle over the divisors at infinity.

The remaining steps in the proof are to show that the map $\Psi$ is injective, onto, and finally a homeomorphism. The proof of injectivity follows an argument of Donaldson based on a function comparing two Hermitian metrics. We show surjectivity by exhibiting a construction which gives a compatible $U(2)$-instanton on the restriction of a vector bundle $\mathcal{E}$ to $\Sigma \times S^{1} \times \mathbf{R} \subset S$, where $\mathcal{E}$ represents an element of $\mathcal{Z}$. This construction is based on Donaldson's theorem that 
any stable bundle on a compact algebraic surface admits a Hermitian-Einstein connection, and on Uhlenbeck compactness for instantons. We take a sequence of Hodge metrics on $S$ which converge to the cylinder metric on compact subsets of $\Sigma \times S^{1} \times \mathbf{R}$. Donaldson's theorem gives a corresponding sequence of Hermitian-Einstein connections on $\mathcal{E}$, which has a weakly convergent subsequence yielding a $U(2)$-instanton $A$ on the cylinder. The stability of $\mathcal{E}$ ensures that no energy is lost in this process and we are able to show that $\Psi([A])=[\mathcal{E}]$.

The last step in the proof is to show that $\Psi$ is a homeomorphism. The key point here (also used in the proof of surjectivity) is that for certain sequences of stable bundles on $S$, convergence on a suitably chosen subset of $S$ actually implies global convergence.

Acknowledgements This paper is a modified version of my Columbia University $\mathrm{PhD}$ thesis. I am very grateful to my advisor, John Morgan, for his invaluable help and advice. Thanks also to Michael Thaddeus, Robert Friedman, Mehrzad Ajoodanian, Pedram Safari and Saso Strle for useful discussions. I am grateful to the referee for pointing out an error in an earlier draft.

\section{Definitions and notation}

We let $Y$ denote the 3 -manifold $\Sigma \times S^{1}$ as above. Fix a Kähler metric $g_{\Sigma}$ on $\Sigma$. Let $\theta$ and $t$ be the standard coordinates on $S^{1}$ and $\mathbf{R}$ respectively. Then

$$
g_{c y l}=g_{\Sigma}+d \theta^{2}+d t^{2}
$$

is a complete Kähler metric on $\Sigma \times S^{1} \times \mathbf{R}$.

The cylinder $\Sigma \times S^{1} \times \mathbf{R}$ embeds in the product ruled surface $S=\Sigma \times \mathbf{C P}^{1}$ in such a way that the standard coordinate $z$ on $\mathbf{C P}^{\mathbf{1}}$ is given by

$$
z=e^{t} e^{i \theta} .
$$

Let $E_{\Sigma}$ denote the rank 2, degree 1 complex vector bundle over $\Sigma$, with a chosen Hermitian metric. Let $E_{Y}$ and $E$ denote the pullbacks of $E_{\Sigma}$ to $Y$ and $Y \times \mathbf{R}$ respectively.

Given a unitary connection $A$ on $E$ we define the energy of $A$ to be

$$
e(A)=\int_{Y \times \mathbf{R}}\left|F_{A}^{0}\right|^{2} \mathrm{dvol}=-\int_{Y \times \mathbf{R}} \operatorname{Tr} F_{A}^{0} \wedge * F_{A}^{0}
$$

where $F_{A}^{0}$ is the trace-free part of the curvature. This is equal to the Yang-Mills energy of the $S O(3)$ connection obtained by projectivising $A$ and $E$. 
Definition 2.1 A $U(2)$-instanton on $E$ is a finite-energy projectively antiself-dual connection on $E$, with fixed central part. That is, a unitary connection $A$, which with respect to the metric $g_{c y l}$, has

- trace-free part of curvature $F_{A}^{0}$ is anti-self-dual

- finite energy

- fixed central part.

For convenience, we choose the fixed central part to be pulled back from $\Sigma$, with $\operatorname{Tr} F_{A}$ harmonic.

We denote by $\mathcal{M}$ the space of $U(2)$-instantons modulo gauge transformations which fix the central part. We denote by $[A]$ the element of $\mathcal{M}$ represented by a $U(2)$-instanton $A$. Let $\mathcal{M}_{e}$ denote the set of equivalence classes with energy a fixed real number $e$. For $e<0, \mathcal{M}_{e}$ is empty; for $e=0, \mathcal{M}_{e}$ consists of equivalence classes of flat connections. We will see in Section 3 that $e$ is always an integer multiple of $4 \pi^{2}$.

The definition of $U(2)$-instanton specifies that the central part of the connection is fixed and pulled back from $\Sigma$. This then determines a holomorphic structure on the degree 1 smooth complex line bundle on $\Sigma$; we denote this holomorphic line bundle by $\mathcal{L}$. We define $\mathcal{D}$ to be the line bundle on $S$ determined by $\mathcal{L}$ and a number $e \in 4 \pi^{2} \mathbf{Z}$ as follows:

$$
\mathcal{D}= \begin{cases}\pi_{1}^{*} \mathcal{L} & \text { if } e(A) \in 8 \pi^{2} \mathbf{Z} \\ \pi_{1}^{*} \mathcal{L} \otimes \pi_{2}^{*} \mathcal{O}_{\mathbf{C P}^{1}}(1) & \text { otherwise }\end{cases}
$$

We also define an integer $c$, again determined by $\mathcal{L}, e$ :

$$
c=\frac{1}{4} c_{1}(\mathcal{D})^{2}+\frac{1}{8 \pi^{2}} e(A) .
$$

Definition 2.2 Fix a line bundle $\mathcal{D} \rightarrow S$ and an integer $c$. Let $\mathcal{Z}_{(\mathcal{D}, c)}$ denote the set of isomorphism classes of rank 2 holomorphic bundles $\mathcal{E}$ on $S$ satisfying

- $\operatorname{det}(\mathcal{E}) \cong \mathcal{D}$

- $c_{2}(\mathcal{E})=c$

- the restriction of $\mathcal{E}$ to $\Sigma_{0}, \Sigma_{\infty}$ is stable.

For a given bundle $\mathcal{E}$ as above, let $[\mathcal{E}]$ be the element of $\mathcal{Z}_{(\mathcal{D}, c)}$ it represents. 


\section{$3 \quad$ Finite energy instantons on the cylinder}

We start by studying the moduli space $\mathcal{M}$ of $U(2)$-instantons on $Y \times \mathbf{R}$ (defined in Section 2). The topology on this space is as follows: a sequence $\left[A_{n}\right]$ in $\mathcal{M}$ converges to a limit $\left[A_{\infty}\right]$, if and only if for some representatives, $A_{n}$ converges to $A_{\infty}$ in $C^{\infty}$ on compact subsets of $Y \times \mathbf{R}$.

Remark 3.1 Throughout this paper (unless explicitly stated otherwise) unitary gauge equivalence refers only to gauge transformations which fix the central part. This is actually equivalent to considering the group of even $S O(3)$ gauge transformations (see [2]).

Projectively flat connections on $E_{Y} \rightarrow Y$ are unitary connections with a fixed central part whose curvature is central. The moduli space of projectively flat connections on $E_{Y}$ modulo gauge is denoted by $\mathcal{R}$. It consists of two connected components $\mathcal{R}_{+}$and $\mathcal{R}_{-}$, each of which is a copy of the moduli space of projectively flat connections on $E_{\Sigma}$. By the Narasimhan-Seshadri theorem this moduli space is diffeomorphic to the moduli space $\mathcal{N}(\Sigma)$ of rank 2 , degree 1 stable bundles over $\Sigma$ (with fixed determinant). It is a smooth compact complex manifold of complex dimension $3 g-3$. The sets $\mathcal{R}_{+}$and $\mathcal{R}_{-}$consist of those projectively flat connections whose holonomy around the $S^{1}$ factor is $+\mathbf{1}$ and $\mathbf{- 1}$ respectively.

We now recall some results about finite energy instantons on cylindrical end manifolds.

Let $A$ be a $U(2)$-instanton on $E \rightarrow Y \times \mathbf{R}$. Fixing a bundle isomorphism

$$
\eta: E_{Y} \times \mathbf{R} \rightarrow E,
$$

the pullback $\eta^{*} A$ gives a 1 -parameter family of unitary connections on $E_{Y}$, which we denote by $A_{t}$. We will need to use the following results about the behaviour of the gauge equivalence class $\left[A_{t}\right]$ :

Theorem 3.2 The class $\left[A_{t}\right]$ converges to a projectively flat connection $\left[A_{ \pm \infty}\right]$ on $Y$ as $t \rightarrow \pm \infty$.

Theorem 3.3 For any gauge representatives $A_{ \pm \infty}$ of the limits of $\left[A_{t}\right]$, there are numbers $C, \delta>0$ and smooth gauge transformations $g_{ \pm}$such that the following estimates hold:

$$
\left\|g_{ \pm}^{*} A-\pi_{Y}^{*} A_{ \pm \infty}\right\|_{L_{2}^{2}\left(Y \times\left[T-\frac{1}{2}, T+\frac{1}{2}\right]\right)} \leq C e^{\mp \delta T} \quad \text { for } \pm T>>0 .
$$


The first of these results follows from Theorem 4.0.1 of [15], with $G=S O(3)$ (note that $\mathcal{M}$ may equivalently be described as the space of finite-energy $S O(3)$ instantons on the projectivisation of $E$, modulo gauge transformations which lift to $S U(2)$ ). The second follows from Theorem 5.2.2 of [15] (see also Lemma 2.1 .10 in $[14])$.

If $A$ is a $U(2)$-instanton converging to projectively flat limits $A_{ \pm \infty}$ as in Theorem 3.2, then the energy of $A$ is related to the value of the Chern-Simons functional on the limits as follows:

$$
\frac{1}{8 \pi^{2}} e(A)=\frac{1}{8 \pi^{2}} \int_{Y \times \mathbf{R}} \operatorname{Tr} F_{A}^{0} \wedge F_{A}^{0}=\left(\mathbf{c s}\left(A_{+\infty}\right)-\mathbf{c s}\left(A_{-\infty}\right)\right) \bmod \mathbf{Z} .
$$

(In fact the second equality is the definition of the Chern-Simons functional, which is $\mathbf{R} / \mathbf{Z}$-valued and is only defined up to an additive constant.)

Given a projectively flat connection $a$ on $\Sigma$, the pullback $\pi_{\Sigma}^{*} a$ represents an element of $\mathcal{R}_{+}$. Trivialise the degree one $U(2)$ bundle over a disk $D \subset \Sigma$ and over its complement, with transition function

$$
\left(\begin{array}{cc}
e^{i \phi} & 0 \\
0 & 1
\end{array}\right)
$$

along the boundary circle. Then $g=\left(\begin{array}{cc}e^{-i \theta} & 0 \\ 0 & 1\end{array}\right)$ is a well-defined unitary gauge transformation over $Y$.

Then the connection

$$
\mathcal{I}_{Y}\left(\pi_{\Sigma}^{*} a\right)=g^{*}\left(\pi_{\Sigma}^{*} a+\frac{i}{2} d \theta \cdot \mathbf{1}_{E_{Y}}\right)
$$

represents an element of $\mathcal{R}_{-}$(Adding the term $\frac{i}{2} d \theta \cdot \mathbf{1}_{E_{Y}}$ gives the desired holonomy around the $S^{1}$ factor, the gauge transformation is required so that elements of $\mathcal{R}_{+}$and $\mathcal{R}_{-}$have the same central part.) Note that $g$ is not in the restricted gauge group of unitary gauge transformations which fix the central part, but that the square of $\mathcal{I}_{Y}$ is equivalent to applying the gauge transformation $e^{i \theta} g^{2}$, which is.

The given map $\mathcal{I}_{Y}$ is thus an involution on $\mathcal{R}$ which interchanges the components $\mathcal{R}_{+}$and $\mathcal{R}_{-}$. It also changes the value of cs by $\frac{1}{2}$ (see $[2,8]$ ). It follows that the energy $e(A)$ is an integer multiple of $4 \pi^{2}$. Also $\frac{1}{4 \pi^{2}} e(A)$ is even if the limits $A_{ \pm \infty}$ are in the same component, odd otherwise.

Let $A \in \mathcal{M}_{e}$ for some $e \in 4 \pi^{2} \mathbf{Z}$. Then $A$ defines a type $(0,1)$ operator $\bar{\partial}_{A}$ on $E$-valued forms which satisfies $\bar{\partial}_{A}^{2}=0$. The Newlander-Nirenberg theorem 
implies that $\bar{\partial}_{A}$ defines a holomorphic structure on the complex vector bundle $E$ (see eg [7, page 46]).

We will now give the definition of an involution

$$
\mathcal{I}: \mathcal{M}_{e} \rightarrow \mathcal{M}_{e}
$$

and show that $\left(E, \bar{\partial}_{A}\right) \cong\left(E, \bar{\partial}_{\mathcal{I}(A)}\right)$ as holomorphic bundles.

Lemma 3.4 There is an involution $\mathcal{I}: \mathcal{M}_{e} \rightarrow \mathcal{M}_{e}$ which fixes the holomorphic structure on $E$ determined by a $U(2)$-instanton. Its restriction to $Y$ is the involution $\mathcal{I}_{Y}$ which switches the components $\mathcal{R}_{+}, \mathcal{R}_{-}$of the space $\mathcal{R}$ of projectively flat connections.

Proof We define the map $\mathcal{I}$ by

$$
A \mapsto g^{*}\left(A+\frac{i}{2} d \theta \cdot \mathbf{1}_{E}\right),
$$

where $g$ is a $U(2)$ gauge transformation to fix the central part as required by the definition of $U(2)$-instanton. (For example take $g=\left(\begin{array}{cc}e^{-i \theta} & 0 \\ 0 & 1\end{array}\right)$ as above).

It is fairly clear that this defines an involution on $\mathcal{M}$ whose restriction to $Y$ is the involution $\mathcal{I}_{Y}$. It also clearly preserves the energy.

It remains to see that it preserves the holomorphic structure. This follows from the $\bar{\partial}$-Poincaré lemma on $\mathbf{C}-\{0\}$, applied to the $(0,1)$ part of $\frac{i}{2} d \theta$. This shows that the map

$$
A \mapsto A+\frac{i}{2} d \theta \cdot \mathbf{1}_{E}
$$

is given by a (central) complex gauge transformation.

(In fact taking $h=|z|^{-\frac{1}{2}}$ yields $h^{-1} \bar{\partial} h=\frac{i}{2} d \theta^{(0,1)}$, as the reader may verify.)

Using Theorem 3.3 and Lemma 3.4 we can modify a $U(2)$-instanton in order to get good gauge representatives on each end of the cylinder.

Lemma 3.5 Let $A$ be a $U(2)$-instanton on $E \rightarrow \Sigma \times S^{1} \times \mathbf{R}$ with energy $e(A)=e \in 4 \pi^{2} \mathbf{Z}$. Then there exists a projectively ASD connection $\tilde{A}$ on $E \rightarrow$ $\Sigma \times S^{1} \times \mathbf{R}$ with the following properties:

- $\left(E, \bar{\partial}_{A}\right) \cong\left(E, \bar{\partial}_{\tilde{A}}\right)$ as holomorphic bundles 
- $e(A)=e(\tilde{A})$

- the limits $\left[\tilde{A}_{ \pm \infty}\right]$ on $Y$ are gauge equivalent (under the full $U(2)$ gauge group) to the pullbacks $\pi_{\Sigma}^{*} a_{ \pm}$of projectively flat connections $a_{ \pm}$on $\Sigma$.

Proof There are 4 cases to consider, depending on whether the limits $\left[A_{ \pm \infty}\right]$ are in $\mathcal{R}_{+}$or $\mathcal{R}_{-}$.

The first two cases occur when both limits $\left[A_{ \pm \infty}\right]$ are in the same component of $\mathcal{R}$; this occurs when $\frac{e}{4 \pi^{2}}$ is even.

If both of $\left[A_{ \pm \infty}\right]$ are in $\mathcal{R}_{+}$, then simply take $\tilde{A}=A$.

If both of $\left[A_{ \pm \infty}\right]$ are in $\mathcal{R}_{-}$, then take $\tilde{A}=\mathcal{I}(A)$.

The other two cases occur when $A$ has "mixed limits", ie, when $\frac{e}{4 \pi^{2}}$ is odd.

Suppose $\left[A_{-\infty}\right] \in \mathcal{R}_{+}$and $\left[A_{+\infty}\right] \in \mathcal{R}_{-}$. Then $\left[A_{-\infty}\right]$ is represented by the pullback $\pi_{\Sigma}^{*} a_{-\infty}$ of a projectively flat connection $a_{-\infty}$ on $\Sigma$. The limit $\left[A_{+\infty}\right]$ is represented by

$$
A_{+\infty}=g^{*}\left(\pi_{\Sigma}^{*} a_{+\infty}+\frac{i}{2} d \theta \cdot \mathbf{1}_{E}\right)=g^{*}\left(\pi_{\Sigma}^{*} a_{+\infty}\right)+\frac{i}{2} d \theta \cdot \mathbf{1}_{E},
$$

with $g$ as in the proof of Lemma 3.4 and $a_{+\infty}$ a projectively flat connection on $\Sigma$.

Define

$$
\tilde{A}=A-s(t) \frac{i}{2} d \theta \cdot \mathbf{1}_{E}
$$

where $s(t)$ is a smooth function on $\mathbf{R}$ with

$$
s(t)= \begin{cases}0 & \text { if } t<0 \\ 1 & \text { if } t>1\end{cases}
$$

This $\tilde{A}$ satisfies the required conditions. It is projectively ASD with the same energy as $A$ since its trace-free part is the same as that of $A$. The holomorphic bundle $\left(E, \bar{\partial}_{\tilde{A}}\right)$ is equal to $\left(E, \bar{\partial}_{A}\right)$ tensored by a line bundle pulled back from $\mathbf{C}^{*}$; any such line bundle is holomorphically trivial. By construction the limits $\left[\tilde{A}_{ \pm \infty}\right]$ have the required property.

If $\left[A_{-\infty}\right] \in \mathcal{R}_{-}$and $\left[A_{+\infty}\right] \in \mathcal{R}_{+}$, define $\tilde{A}=\widetilde{\mathcal{I}(A)}$.

Note that if $[A],[B] \in \mathcal{M}_{e}$ then $\tilde{A}$ is gauge equivalent to $\tilde{B}$ by a unitary gauge transformation fixing the central part if and only if $[A]=[B]$ or $[A]=$ $\mathcal{I}([B])$. 
Remark 3.6 The preceding Lemma may be considered from the point of view of orbifold bundles and connections. A connection on $\Sigma \times S^{1} \times \mathbf{R}$ which is converging to a flat connection with holonomy $\mathbf{- 1}$ around the $S^{1}$ factor will clearly not extend over $\Sigma \times \mathbf{C P}^{\mathbf{1}}$. We may think of such a connection as a connection on the orbifold $\Sigma \times \mathbf{C P}^{\mathbf{1}}$ with $\mathbf{Z}_{2}$ acting on $\mathbf{C P}^{\mathbf{1}}$ by $z \mapsto z^{2}$. We can then produce a connection with $+\mathbf{1}$ holonomy by tensoring with an orbifold line bundle connection with matching holonomy limits.

\section{Stable bundles on $\Sigma$}

In this section we briefly note two very important properties of the moduli space $\mathcal{N}(\Sigma)$ of rank 2 , degree 1 stable bundles on $\Sigma$ with fixed determinant. For a detailed study of this space see [1], [16].

Firstly, $\mathcal{N}(\Sigma)$ is a fine moduli space (see [1, Section 9],[16] for a proof, also [10, Section 4.2] for general discussion). What this means is that there is a holomorphic bundle $\mathcal{U}$ over $\Sigma \times \mathcal{N}(\Sigma)$ with the following universal property. Let $M$ be any complex manifold. For any holomorphic bundle $\mathcal{V}$ over $\Sigma \times M$, whose restriction to each $\Sigma \times\{$ point $\}$ is stable, there exists a unique holomorphic map $f: M \rightarrow \mathcal{N}(\Sigma)$ and a line bundle $\mathcal{H}$ on $M$ such that $\mathcal{V} \cong(\operatorname{Id} \times f)^{*} \mathcal{U} \otimes \pi_{2}^{*} \mathcal{H}$.

Secondly, there are local slices for the action of the complex gauge group on the space of stable $(0,1)$ connections on $E_{\Sigma} \rightarrow \Sigma$. This means the following. Let $\mathcal{A}_{0}^{(0,1)}$ denote the space of all $(0,1)$ connections on $E_{\Sigma}$ with fixed central part, and let $\mathcal{G}_{0}^{c}=\Gamma\left(S L\left(E_{\Sigma}\right)\right)$ denote the group of complex gauge automorphisms fixing the central part. (In fact for the following discussion we take the $L_{1}^{2}$ completion of $\mathcal{A}_{0}^{(0,1)}$ and the $L_{2}^{2}$ completion of the complex gauge group.) The group $\mathcal{G}_{0}^{c}$ acts on $\mathcal{A}_{0}^{(0,1)}$ with stabiliser $\pm \mathbf{1}$. The set of all holomorphic structures on $E_{\Sigma}$ is the quotient of $\mathcal{A}_{0}^{(0,1)}$ by this action. This space is not Hausdorff. If we let $\mathcal{A}^{s} \subset \mathcal{A}_{0}^{(0,1)}$ denote the subset of stable holomorphic structures, then the quotient of $\mathcal{A}^{s}$ by $\mathcal{G}_{0}^{c}$ is the moduli space $\mathcal{N}(\Sigma)$.

The differential at any point $\bar{\partial}_{0} \in \mathcal{A}_{0}^{(0,1)}$ of the complex gauge group action is given by the operator

$$
\bar{\partial}_{0}: \Omega^{0}\left(\Sigma, \text { End } E_{\Sigma}\right) \rightarrow \Omega^{0,1}\left(\Sigma, \text { End } E_{\Sigma}\right)
$$

(restricted to trace-free forms). The metrics on $\Sigma$ and $E_{\Sigma}$ enable us to define a formal adjoint $\bar{\partial}_{0}^{*}$. Denote by

$$
S_{\bar{\partial}_{0}}=\bar{\partial}_{0}+\operatorname{Ker} \bar{\partial}_{0}^{*}
$$


the affine slice in $\mathcal{A}_{0}^{(0,1)}$. This is an affine complex subspace which is transverse to the gauge orbit at the point $\bar{\partial}_{0}$. Suppose that $\bar{\partial}_{0}$ gives a stable holomorphic structure. The action of $\mathcal{G}_{0}^{c} /\{ \pm \mathbf{1}\}$ on $\mathcal{A}^{s}$ is smooth, free and proper; it follows that there is a neighbourhood $U$ of $\bar{\partial}_{0}$ in $S_{\bar{\partial}_{0}}$, such that the natural map

$$
U \times_{ \pm 1} \mathcal{G}_{0}^{c} \rightarrow \mathcal{A}_{0}^{(0,1)}
$$

is a diffeomorphism onto its image. (See [10, pages 294-295 and page 300] for details. The discussion there is for a base manifold of any dimension; the dimension one case is simpler since every $(0,1)$ connection is integrable.)

\section{$5 \quad$ Stable bundles on $S$}

In this section we note some facts about stable bundles on the surface $S=\Sigma \times \mathbf{C P}^{\mathbf{1}}$, and in particular about the set $\mathcal{Z}_{(\mathcal{D}, c)}$ defined in Section 2 .

Let $\sigma$ denote the Poincaré dual of the homology class of $S$ represented by $\Sigma \times\{$ point $\}$, and $f$ the dual of the class represented by $\{$ point $\} \times \mathbf{C P}^{\mathbf{1}}$. Then $H^{2}(S, \mathbf{R})=H^{(1,1)}(S, \mathbf{R})$ is generated by the classes $\sigma$ and $f$. The intersection pairing gives

$$
\sigma \cdot \sigma=f \cdot f=0, \quad \sigma \cdot f=1 .
$$

The Kähler cone $\mathcal{K}(S)$ is the quadrant $\{a \sigma+b f \mid a, b>0\}$; any such class is represented by the associated $(1,1)$-form of some Kähler metric on $S$.

We now recall the definition of stability in the sense of Mumford and Takemoto. Let $X$ be a complex manifold of dimension $n$. Let $[\omega] \in H^{(1,1)}(X, \mathbf{R})$ be an element of $\mathcal{K}(X)$. For any torsion-free coherent sheaf $\xi$ over $X$ we set

$$
\mu(\xi)=c_{1}(\xi) \cdot[\omega]^{n-1} / \operatorname{rank} \xi .
$$

The sheaf $\xi$ is $[\omega]$-slope stable (resp. semistable) if for any proper coherent subsheaf $\zeta$ of $\xi$ we have

$$
\mu(\zeta)<\mu(\xi) \quad(\text { resp. } \quad \mu(\zeta) \leq \mu(\xi)) .
$$

For rank 2 sheaves on a surface, stability with respect to two different elements of the Kähler cone is equivalent if and only if they represent points in the same chamber (see [9, page 142], [17]). The chamber structure depends on the Chern classes of the sheaves in question. Any choice of $c_{1}, c_{2}$ determines a finite set of walls, in the Kähler cone $\mathcal{K}$, as follows: any $\zeta \in H^{(1,1)}(X, \mathbf{R})$ satisfying

$$
\left\{\begin{array}{c}
\zeta=c_{1} \quad(\bmod 2) \\
c_{1}^{2}-4 c_{2} \leq \zeta^{2}<0
\end{array}\right\}
$$


determines a wall $\zeta^{\perp}=\{x \in \mathcal{K} \mid x \cdot \zeta=0\}$. Denote by $\mathcal{W}$ the union of all such walls.

The chambers are then the connected components of $\mathcal{K}-\mathcal{W}$. In our case the Kähler cone is just the first quadrant in $H^{(1,1)}(S, \mathbf{R}) \cong \mathbf{R}^{2}$, and the walls are rays with positive slope. See Figure 1 for the chamber structure in $\mathcal{K}(S)$ for some choices of $\left(c_{1}, c_{2}\right)$. Note that as $4 c_{2}-c_{1}^{2}$ increases, so does the number of walls.
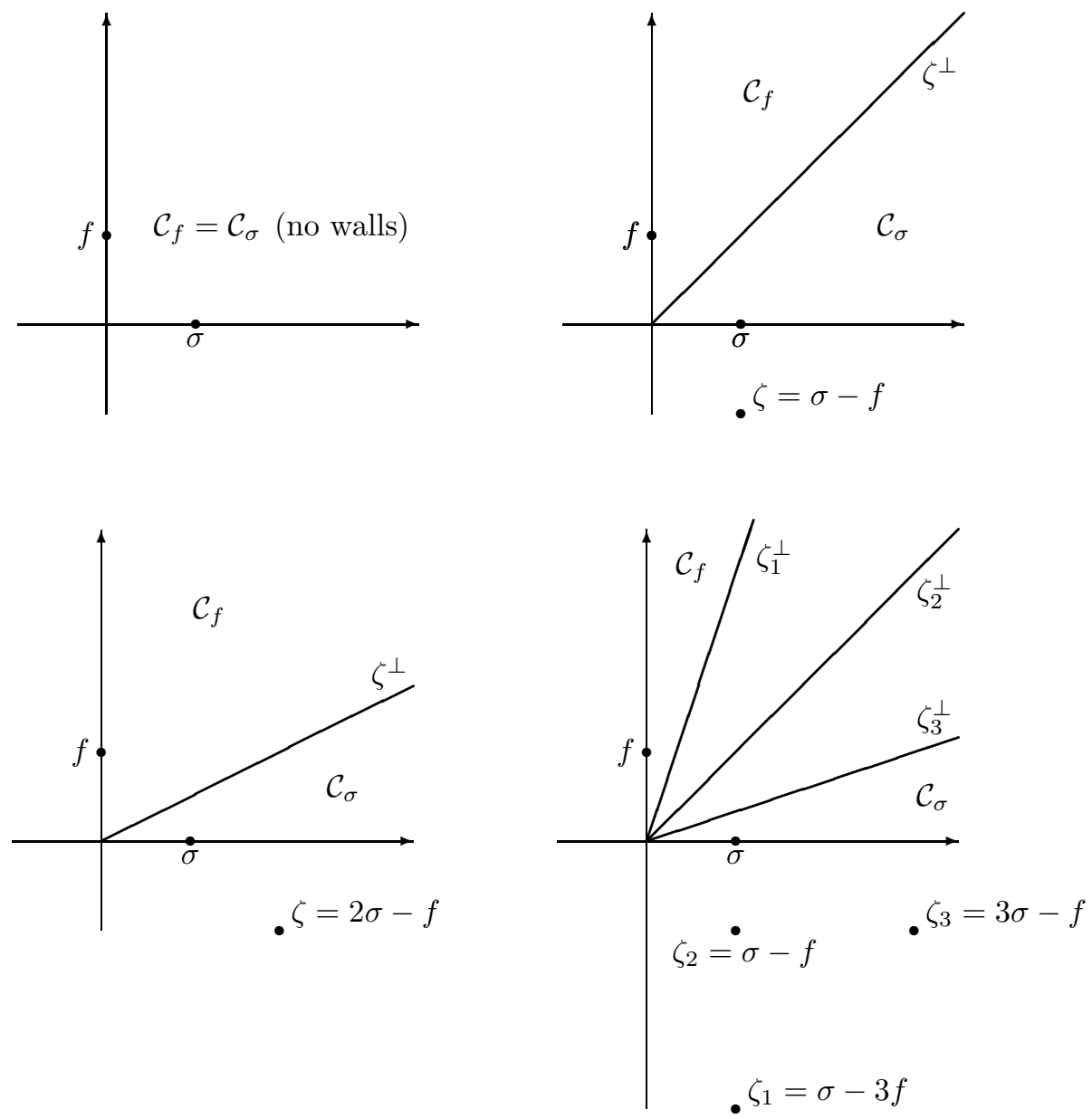

Figure 1: Chamber Structure for $\left(c_{1}, c_{2}\right)=(f, 0),(\sigma+f, 1),(f, 1),(\sigma+f, 2)$

We will be concerned with the chamber $\mathcal{C}_{\sigma}$, whose boundary contains the point $\sigma$. A bundle or sheaf is said to be $\mathcal{C}_{\sigma}$-stable if it is $[\omega]$-stable for any $[\omega] \in \mathcal{C}_{\sigma}$. 
Observe that for any fixed choice of $\left(c_{1}, c_{2}\right)$, the point $n \sigma+f$ will be in $\mathcal{C}_{\sigma}$ for large enough $n$.

Fix some line bundle $\mathcal{D}$ on $S$ with $\sigma \cdot c_{1}(\mathcal{D})=1$ and some integer $c$. Then we denote by $\mathcal{M}_{(\mathcal{D}, c)}$ the set of isomorphism classes of $\mathcal{C}_{\sigma}$-stable bundles with determinant and second Chern class given by $\mathcal{D}$ and $c$ respectively.

For any choice of $\mathcal{D}, c$, let $\tilde{E} \rightarrow S$ be a smooth rank two complex vector bundle with $c_{1}(\tilde{E})=c_{1}(\mathcal{D})$ and $c_{2}(\tilde{E})=c$. Let $\mathcal{A}^{(0,1)}(\tilde{E})$ denote the space of all integrable $(0,1)$ connections on $\tilde{E}$ (with the $C^{\infty}$ topology) and let $\mathcal{G}^{c}$ denote the group of complex linear bundle automorphisms of $\tilde{E}$. Then we topologise $\mathcal{M}_{(\mathcal{D}, c)}$ as a subspace of $\mathcal{A}^{(0,1)}(\tilde{E}) / \mathcal{G}^{c}$. That is to say, a sequence of elements $\left[\mathcal{E}_{n}\right]$ of $\mathcal{M}_{(\mathcal{D}, c)}$ converge to a limit $\left[\mathcal{E}_{\infty}\right]$ if and only if some sequence of $(0,1)$ connections $\bar{\partial}_{n}$ on $\tilde{E}$ representing $\left[\mathcal{E}_{n}\right]$ converge in $C^{\infty}$ to a limit $\bar{\partial}_{\infty}$ which represents $\left[\mathcal{E}_{\infty}\right]$.

We will often use the term " $\bar{\partial}$-operator" to describe integrable $(0,1)$ connections.

The following facts about $\mathcal{C}_{\sigma}$-stable bundles follow immediately from [9, Chapter 6 , Theorem 5] and the fact that a rank 2, odd degree semistable bundle on $\Sigma$ must be stable.

Proposition 5.1 Let $\mathcal{E} \rightarrow S$ be a rank 2 holomorphic vector bundle with $\operatorname{det}(\mathcal{E})=\mathcal{D}$ and $c_{2}(\mathcal{E})=c$.

- If $\mathcal{E}$ is $\mathcal{C}_{\sigma}$-stable then its restriction to $\Sigma \times\{z\}$ is stable for all but finitely many $z \in \mathbf{C P}^{\mathbf{1}}$.

- If the restriction of $\mathcal{E}$ to $\Sigma \times\{z\}$ is stable for some $z \in \mathbf{C P}^{\mathbf{1}}$ then $\mathcal{E}$ is $\mathcal{C}_{\sigma}$-stable.

Note it follows that $\mathcal{Z}_{(\mathcal{D}, c)}$ is a subset of $\mathcal{M}_{(\mathcal{D}, c)}$.

Given a bundle $\mathcal{E}$ with $[\mathcal{E}] \in \mathcal{M}_{(\mathcal{D}, c)}$ which is unstable on $\Sigma \times\left\{z_{i}\right\}$ for some finite set $z_{1}, \ldots, z_{k} \in \mathbf{C P}^{\mathbf{1}}$, one may apply a finite sequence of elementary modifications to obtain a bundle $\mathcal{E}^{\prime}$ which is stable on $\Sigma \times\{z\}$ for all $z \in \mathbf{C P}^{\mathbf{1}}$, and such that

$$
\left.\left.\mathcal{E}\right|_{\Sigma \times\left(\mathbf{C P}^{1}-\cup z_{i}\right)} \cong \mathcal{E}^{\prime}\right|_{\Sigma \times\left(\mathbf{C P}^{1}-\cup z_{i}\right)} .
$$

(See [9, pages 41, 98, 148].)

For any $z \in \mathbf{C P}^{\mathbf{1}}$,

$$
\operatorname{det}\left(\left.\mathcal{E}\right|_{\Sigma \times\{z\}}\right)=\left.\mathcal{D}\right|_{\Sigma \times\{z\}}
$$


This gives the same element of Pic $\Sigma$ for all $z$. Thus for each $z \in \mathbf{C P}^{\mathbf{1}}-\cup z_{i}$, the restriction $\left.\mathcal{E}\right|_{\Sigma \times\{z\}}$ determines a point in the moduli space $\mathcal{N}(\Sigma)$. This implies (see Section 4) that in fact $[\mathcal{E}]$ determines a holomorphic map

$$
f_{\mathcal{E}}: \mathbf{C P}^{1}-\cup z_{i} \rightarrow \mathcal{N}(\Sigma) .
$$

Also $\mathcal{E}^{\prime}$ determines an extension of this holomorphic map to all of $\mathbf{C P}^{\mathbf{1}}$, which we still denote by $f_{\mathcal{E}}$.

The following Proposition shows that in fact $[\mathcal{E}] \in \mathcal{M}_{(\mathcal{D}, c)}$ is completely determined by the map $f_{\mathcal{E}}$ and by local information at the curves $\Sigma \times\left\{z_{i}\right\}$ on which $\mathcal{E}$ has unstable restriction.

Proposition 5.2 A $\mathcal{C}_{\sigma}$-stable bundle $\mathcal{E}$ which satisfies

$$
\sigma \cdot c_{1}(\mathcal{E})=1, \quad f \cdot c_{1}(\mathcal{E})=0 \text { or } 1
$$

determines, and is determined up to isomorphism by, a triple $\left(f_{\mathcal{E}}, \cup_{i=1}^{k} z_{i}, \cup_{i=1}^{k}\left[\mathcal{E}_{i}\right]\right)$, where

- $f_{\mathcal{E}}$ is a holomorphic map from $\mathbf{C P}^{\mathbf{1}}$ to $\mathcal{N}(\Sigma)$,

- $z_{1}, \ldots, z_{k}$ are distinct (unordered) points in $\mathbf{C P}^{\mathbf{1}}(k \geq 0)$, and

- $\left[\mathcal{E}_{i}\right]$ is an $f_{\mathcal{E}}$-compatible holomorphic vector bundle over a germ of a neighbourhood of $\Sigma \times\left\{z_{i}\right\}$, ie, an equivalence class of bundles $\mathcal{E}_{i} \rightarrow$ $\Sigma \times D_{i}$ where $D_{i}$ is a small disk neighbourhood of $z_{i}$ in $\mathbf{C P}^{\mathbf{1}},\left.\mathcal{E}_{i}\right|_{\Sigma \times\left\{z_{i}\right\}}$ is unstable, and

$$
\left.\mathcal{E}_{i}\right|_{\Sigma \times\left(D_{i}-z_{i}\right)} \cong\left(\operatorname{Id} \times\left. f_{\mathcal{E}}\right|_{D_{i}-z_{i}}\right)^{*} \mathcal{U} .
$$

Two such bundles $\mathcal{E}_{i} \rightarrow \Sigma \times D_{i}$ and $\mathcal{E}_{i}^{\prime} \rightarrow \Sigma \times D_{i}^{\prime}$ are equivalent if they are isomorphic on $\Sigma \times\left(D_{i} \cap D_{i}^{\prime}\right)$.

Proof It is clear from the previous discussion that a $\mathcal{C}_{\sigma}$-stable bundle determines such a triple. Conversely, suppose we are given $\left(f_{\mathcal{E}}, \cup_{i=1}^{k} z_{i}, \cup_{i=1}^{k}\left[\mathcal{E}_{i}\right]\right)$. We construct a bundle $\mathcal{E}$ over $S$ by gluing together the bundle $\left(\operatorname{Id} \times\left. f_{\mathcal{E}}\right|_{\mathbf{C P}^{1}-\cup z_{i}}\right)^{*} \mathcal{U}$ on $\Sigma \times\left(\mathbf{C P}^{\mathbf{1}}-\cup z_{i}\right)$ and the bundles $\mathcal{E}_{i}$ on $\Sigma \times D_{i}$. The gluing isomorphisms are well-defined up to multiplication by a $\mathbf{C}^{*}$-valued function on each $D_{i}-z_{i}$. Thus the isomorphism class of the glued-up bundle $\mathcal{E}$ is well-defined up to tensoring with a line bundle pulled back from $\mathbf{C P}^{1}$. The requirement that

$$
f \cdot \operatorname{det}(\mathcal{E})=0 \text { or } 1
$$

determines $[\mathcal{E}]$ uniquely. 
Note that a triple $\left(f_{\mathcal{E}}, \cup_{i=1}^{k} z_{i}, \cup_{i=1}^{k}\left[\mathcal{E}_{i}\right]\right)$ corresponding to an element of $\mathcal{M}_{(\mathcal{D}, c)}$ is in the subset $\mathcal{Z}_{(\mathcal{D}, c)}$ if and only if neither of the points $\{0, \infty\}$ is included in $\cup_{i=1}^{k} z_{i}$. Also note that reordering the points $z_{1}, \ldots, z_{n}$ and, correspondingly, the data $\left[\mathcal{E}_{1}\right], \ldots,\left[\mathcal{E}_{n}\right]$ does not change the bundle $\mathcal{E}$.

Let $\mathcal{M}_{(\mathcal{D}, c)}^{\prime}$ denote the set of all triples for which the associated stable bundle has second Chern class $c$ and determinant $\mathcal{D}$. We topologise $\mathcal{M}_{(\mathcal{D}, c)}^{\prime}$ as follows: a sequence of triples $\left(f_{n}, \cup_{i=1}^{k_{n}} z_{i ; n}, \cup_{i=1}^{k_{n}}\left[\mathcal{E}_{i ; n}\right]\right)$ is converging to a limit $\left(f_{\mathcal{E}}, \cup_{i=1}^{k} z_{i}, \cup_{i=1}^{k}\left[\mathcal{E}_{i}\right]\right)$ if and only if

- $f_{n} \rightarrow f_{\mathcal{E}}$ in $C^{\infty}$ on compact subsets of $\mathbf{C P}^{\mathbf{1}}-\cup_{i=1}^{k} z_{i}$

- for any open neighbourhood $D$ of $\cup_{i=1}^{k} z_{i}$, there exists $N$ such that $\cup_{i=1}^{k_{n}} z_{i ; n} \subset D \quad \forall n \geq N$

- for some open neighbourhood $D$ of $\cup_{i=1}^{k} z_{i}$, the isomorphism classes of bundles determined by $f_{n}$ and $\cup_{i=1}^{k_{n}}\left[\mathcal{E}_{i ; n}\right]$ converge on $\Sigma \times D$ to that determined by $f_{\mathcal{E}}$ and $\cup_{i=1}^{k}\left[\mathcal{E}_{i}\right]$. (Convergence here means that a choice of representatives of the isomorphism classes converges in $C^{\infty}$ on compact sets to a representative of the limit.)

Proposition 5.2 gives a correspondence between $\mathcal{M}_{(\mathcal{D}, c)}$ and $\mathcal{M}_{(\mathcal{D}, c)}^{\prime}$. We will see in Section 8 that in fact this is a homeomorphism.

\section{From instantons to stable bundles}

Let $e \in 4 \pi^{2} \mathbf{Z}$ and let $\mathcal{D}, c$ be determined by $e$ as in (1),(2). In this section we define a function

$$
\Psi: \mathcal{M}_{e} / \mathcal{I} \rightarrow \mathcal{Z}_{(\mathcal{D}, c)}
$$

Let $A$ be a $U(2)$-instanton with energy $e$. Denote by $\bar{\partial}_{A}$ the associated $(0,1)$ operator on $E$-valued forms. We will show that the holomorphic bundle $\left(E, \bar{\partial}_{A}\right)$ has a unique extension $\mathcal{E}$ to the compact surface $S$, up to isomorphism, which satisfies

- $\operatorname{det} \mathcal{E}=\mathcal{D}$

- $c_{2}(\mathcal{E})=c$

- $\left.\mathcal{E}\right|_{\Sigma_{0}},\left.\mathcal{E}\right|_{\Sigma_{\infty}}$ are stable.

If $g$ is a gauge transformation then $\left(E, \bar{\partial}_{A}\right) \cong\left(E, \bar{\partial}_{g^{*} A}\right)$ as holomorphic bundles. This shows that the extension gives a function $\Psi$ on $\mathcal{M}_{e}$, defined by

$$
\Psi([A])=[\mathcal{E}] .
$$


Proposition 3.4 shows that $\Psi$ descends to $\mathcal{M}_{e} / \mathcal{I}$.

We prove two propositions in this section. The first shows how to extend the holomorphic vector bundle $\left(E, \bar{\partial}_{A}\right)$ in a unique way over the compact surface $S$. This could be established following the method of Guo [12]; however the product structure of $Y$ and the fact that $\mathcal{N}(\Sigma)$ is a fine moduli space give a much easier proof in our case. The second proposition confirms that the determinant line bundle and the second Chern class of the extended bundle are given by $\mathcal{D}$ and $c$, respectively.

Proposition 6.1 Let $A$ be a (finite energy) $U(2)$-instanton on $E \rightarrow \Sigma \times$ $S^{1} \times \mathbf{R}$. Then the bundle $E$ with the holomorphic structure determined by $A$ extends uniquely to a holomorphic bundle $\mathcal{E}$ over $S=\Sigma \times \mathbf{C P}^{\mathbf{1}}$ whose restriction to $\Sigma_{0}$ and $\Sigma_{\infty}$ is stable and whose restriction to every fiber $\{$ point $\} \times$ $\mathbf{C P}^{1}$ has degree 0 or 1 .

Proof For convenience, we will work with the connection $\tilde{A}$ obtained from $A$ as in Lemma 3.5.

The proof that the bundle extends over the divisors at infinity is the same for each end. We consider the end $\Sigma_{0}$ (corresponding to $t=-\infty$ ).

Fixing a bundle isomorphism between $E$ and $E_{Y} \times \mathbf{R}$ as in Section $3, \tilde{A}$ gives an associated path $\left[\tilde{A}_{t}\right]$ of gauge-equivalence classes of connections on $E_{Y}$. This path converges to projectively flat limits $\left[\tilde{A}_{ \pm \infty}\right]$ as $t \rightarrow \pm \infty$. By applying a bundle automorphism of $E_{Y}$, we may arrange that $\tilde{A}_{-\infty}$ is pulled back from a projectively flat connection $a_{-\infty}$ on $\Sigma$. Since $\left[\tilde{A}_{t}\right] \rightarrow\left[\tilde{A}_{-\infty}\right]$ as $t \rightarrow-\infty$, it follows that the restriction $\left[\tilde{A}_{(\theta, t)}\right]$ to $\left.E\right|_{\Sigma \times(\theta, t)}$ is converging to $\left[a_{-\infty}\right]$. Thus also the restriction of the holomorphic structure determined by $[\tilde{A}]$ to $\left.E\right|_{\Sigma \times(\theta, t)}$ is converging to that given by $\left[a_{-\infty}\right]$.

Now $a_{-\infty}$ is a projectively flat connection on $E_{\Sigma}$; therefore the holomorphic structure it determines is stable. Stability is an open condition on the space of equivalence classes of holomorphic structures. Thus for some $T<<0$, we must have that $\left[\tilde{A}_{(\theta, t)}\right]$ determines a stable holomorphic structure for each $t<T$. Or in other words $\left(E, \bar{\partial}_{\tilde{A}}\right)$ gives a holomorphic bundle on the product of $\Sigma$ times the punctured disk $D_{0}^{*}=\{(\theta, t): t<T\}=\left\{z: 0<|z|<R=e^{T}\right\}$, whose restriction to each $\Sigma \times\{z\}$ is stable with determinant $\mathcal{L}$, i.e an element of the moduli space $\mathcal{N}(\Sigma)$.

Recall from Section 4 that $\mathcal{N}(\Sigma)$ is a fine moduli space. Thus there exists a holomorphic map

$$
f_{0}: D_{0}^{*} \rightarrow \mathcal{N}(\Sigma)
$$


such that

$$
\left.\left(E, \bar{\partial}_{\tilde{A}}\right)\right|_{\Sigma \times D_{0}} \cong\left(\operatorname{Id} \times f_{0}\right)^{*} \mathcal{U} .
$$

(Noting that there are no nontrivial holomorphic line bundles on $D_{0}{ }^{*}$.) Denote the isomorphism between them by $g_{0}$. This is a section of $G L(E)$ over $\Sigma \times D_{0}{ }^{*}$.

The fact that $f_{0}(z)$ is approaching the point in $\mathcal{N}(\Sigma)$ determined by $a_{-\infty}$ as $z \rightarrow 0$ implies by removable singularities that $f_{0}$ can be extended holomorphically to a map of the disk $D_{0}$ by setting $f_{0}(0)=\left[\bar{\partial}_{a_{-\infty}}\right]$. Then $\mathcal{E}_{0}=\left(\operatorname{Id} \times f_{0}\right)^{*} \mathcal{U}$ is a holomorphic bundle on $\Sigma \times D_{0}$ whose restriction to $\Sigma \times D_{0}{ }^{*}$ is isomorphic to $\left(E, \bar{\partial}_{\tilde{A}}\right)$.

In exactly the same way we can find a disk neighbourhood $D_{\infty}$ of $\infty \in \mathbf{C P}^{1}$ and a bundle $\mathcal{E}_{\infty}$ on $\Sigma \times D_{\infty}$ whose restriction to $\Sigma \times D_{\infty}{ }^{*}$ is isomorphic to $\left(E, \bar{\partial}_{\tilde{A}}\right)$ by some $g_{\infty} \in \Gamma\left(\Sigma \times D_{\infty}{ }^{*}, G L(E)\right)$.

Now we can patch together these bundles using the complex gauge transformations $g_{0}, g_{\infty}$ to form a bundle $\mathcal{E}$ on $\Sigma \times \mathbf{C P}^{1}$ which extends the holomorphic bundle $\left(E, \bar{\partial}_{\tilde{A}}\right)$ on the cylinder. This extension is not unique; the universal property of $\mathcal{U} \rightarrow \Sigma \times \mathcal{N}(\Sigma)$ only defines the bundles $\mathcal{E}_{0}, \mathcal{E}_{\infty}$ up to tensoring with a line bundle pulled back from $D_{0}, D_{\infty}$ respectively. Thus the extension $\mathcal{E}$ is unique up to tensoring with a line bundle pulled back from $\mathbf{C P}^{\mathbf{1}}$. The degree of the restriction of $\mathcal{E}$ to a fiber $\left\{\right.$ point $\times \mathbf{C P}^{\mathbf{1}}$ is given by the intersection pairing $c_{1}(\mathcal{E}) \cdot f$. Tensoring with $\pi_{2}^{*} \mathcal{O}_{\mathbf{C P}^{1}}(a)$ will change this intersection by $2 a$; thus there will be a unique extension $\mathcal{E}$ with $\left.\operatorname{deg} \mathcal{E}\right|_{\{\text {point }\} \times \mathbf{C P}^{1}}=0$ or 1 .

Remark 6.2 Proposition 6.1 does not give an extension of the connection $\tilde{A}$ to $S$, or even an extension of the unitary structure on $E$. We just see that the holomorphic bundle extends over $S$. We will see from the proof of the next Proposition that in fact the metric extends continuously to the extended holomorphic bundle. This is reminiscent of Guo's results [12, I, Theorem 6.1].

Proposition 6.3 Let $A$ be a $U(2)$-instanton on $E \rightarrow \Sigma \times S^{1} \times \mathbf{R}$ with energy $e(A)=e \in 4 \pi^{2} \mathbf{Z}$. Let $\mathcal{E}$ be the extension of the holomorphic bundle $\left(E, \bar{\partial}_{A}\right)$ over $S$ given in Proposition 6.1, and let $\mathcal{D}, c$ be given by (1), (2) in Section 2. Then the determinant line bundle of $\mathcal{E}$ is isomorphic to $\mathcal{D}$ and $c_{2}(\mathcal{E})=c$.

Proof We first consider the determinant line bundle $\operatorname{det} \mathcal{E}$. Note that the central part of the connection $A$ and the central parts of the connections $A_{ \pm \infty}$ and $a_{ \pm \infty}$ described in the proof of Proposition 6.1 are all given by the same fixed central connection pulled back from $\Sigma$. This connection determines a degree 1 holomorphic line bundle $\mathcal{L}$ on $\Sigma$; it follows that the restriction of $\mathcal{E}$ 
to each fiber $\Sigma \times\{$ point $\}$ has $\mathcal{L}$ as its determinant line bundle. This then implies that $\operatorname{det} \mathcal{E} \cong \pi_{1}^{*} \mathcal{L}+\pi_{2}^{*} \mathcal{O}_{\mathbf{C P}^{1}}(a)$ for some $a \in \mathbf{Z}$. The specific choice of the extension $\mathcal{E}$ implies that $a=0$ or 1 . It follows that $c_{1}(\mathcal{E})=a \sigma+f$, so that $c_{1}(\mathcal{E})^{2}=2 a$. The result will now follow if we can establish the following identity:

$$
c_{1}(\mathcal{E})^{2}-4 c_{2}(\mathcal{E})=-\frac{1}{2 \pi^{2}} e(A)
$$

To see this note first that, by (3)

$$
c_{1}(\mathcal{E})^{2}=2 a=-\frac{1}{2 \pi^{2}} e(A) \bmod 4
$$

which implies that

$$
a= \begin{cases}0 & \text { if } e(A) \in 8 \pi^{2} \mathbf{Z}, \\ 1 & \text { otherwise. }\end{cases}
$$

This tells us that $\operatorname{det} \mathcal{E}=\mathcal{D}$ as required; it then follows immediately from (3) that $c_{2}(\mathcal{E})=c$.

We will establish Equation (3) using Chern-Weil theory. For any smooth connection $A^{\prime}$ on a rank 2 complex vector bundle $\mathcal{E}^{\prime}$ over a compact Riemannian 4 -manifold $X$ we have the following identity:

$$
p_{1}\left(\operatorname{ad} \mathcal{E}^{\prime}\right)=c_{1}\left(\mathcal{E}^{\prime}\right)^{2}-4 c_{2}\left(\mathcal{E}^{\prime}\right)=-\frac{1}{2 \pi^{2}} \int_{X} \operatorname{Tr} F_{A^{\prime}}^{0} \wedge F_{A^{\prime}}^{0}
$$

This number is always an integer, and it only depends on the toplogical type of the bundle $\mathcal{E}^{\prime}$. Thus to prove (3) it suffices to find a complex bundle $\mathcal{E}^{\prime}$ which is topologically equivalent to $\mathcal{E}$, and a connection $A^{\prime}$ on $\mathcal{E}^{\prime}$ such that the integral $\int_{X} \operatorname{Tr} F_{A^{\prime}}^{0} \wedge F_{A^{\prime}}^{0}$ differs from $e(A)$ by less than $2 \pi^{2}$. We will do this by gluing together the connection $\tilde{A}$ on the cylinder and flat connections on the divisors at infinity, where $\tilde{A}$ is obtained from $A$ as in Lemma 3.5.

From the proof of Proposition 6.1 the bundle $\mathcal{E}$ is formed by patching together bundles on the cylinder and on neighbourhoods of the divisors at infinity as follows:

$$
\mathcal{E}=\mathcal{E}_{0} \cup_{g_{0}}\left(E, \bar{\partial}_{\tilde{A}}\right) \cup_{g_{\infty}} \mathcal{E}_{\infty},
$$

where $g_{0}, g_{\infty}$ are sections of $G L(E)$ over $\Sigma \times D_{0}{ }^{*}, \Sigma \times D_{\infty}{ }^{*}$ respectively.

Denote by $E_{0}, E_{\infty}$ the smooth hermitian vector bundles underlying $\mathcal{E}_{0}, \mathcal{E}_{\infty}$. Both of these vector bundles are smoothly bundle isomorphic to the pullback of the degree 1 hermitian vector bundle $E_{\Sigma}$ on $\Sigma$.

We again restrict our attention to one end. Without loss of generality, we consider the end corresponding to $z=0$ in $\mathbf{C P}^{\mathbf{1}}$ (ie $t \rightarrow-\infty$ ). After a unitary 
gauge change, we may suppose that $\tilde{A}$ is converging exponentially fast to a limit $\pi_{\Sigma}^{*} a_{-\infty}$, where $a_{-\infty}$ is a projectively flat connection on $\Sigma$. (See Lemma 3.5.)

Let $\bar{\partial}_{\tilde{A}}$ denote the $(0,1)$ operator associated to $\tilde{A}$, and let $\bar{\partial}_{a_{-\infty}}$ be the $(0,1)$ operator on $\Sigma$ associated to $a_{-\infty}$. It follows that the restriction $\left.\bar{\partial}_{\tilde{A}}\right|_{\Sigma_{z}}$ is converging to $\bar{\partial}_{a_{-\infty}}$ as $z \rightarrow 0$. (Here $\Sigma_{z}$ denotes the curve $\Sigma \times\{z\} \subset \Sigma \times \mathbf{C P}^{\mathbf{1}}$.)

Denote by $\bar{\partial}_{B}$ the $(0,1)$ operator on $E_{0} \rightarrow \Sigma \times D_{0}$ which gives the holomorphic structure on $\mathcal{E}_{0}$. Then

$$
\bar{\partial}_{B}=g_{0}^{-1} \circ \bar{\partial}_{\tilde{A}} \circ g_{0} .
$$

The restriction of $\bar{\partial}_{B}$ to $\Sigma_{0}$ is by construction isomorphic to $\bar{\partial}_{a_{-\infty}}$. Thus after possibly changing $\bar{\partial}_{B}$ and $g_{0}$ by a complex gauge automorphism pulled back from $\Sigma$ we may assume that $\left.\bar{\partial}_{B}\right|_{\Sigma_{0}}=\bar{\partial}_{a_{-\infty}}$. Thus we have

$$
\left.\bar{\partial}_{\tilde{A}}\right|_{z} \rightarrow \bar{\partial}_{a_{-\infty}} \quad \text { as } z \rightarrow 0,
$$

and also

$$
\left.g_{0}^{-1} \circ \bar{\partial}_{\tilde{A}} \circ g_{0}\right|_{\Sigma_{z}} \rightarrow \bar{\partial}_{a_{-\infty}} \quad \text { as } z \rightarrow 0 .
$$

We may also assume that the central part of $\bar{\partial}_{B}$ is pulled back from $\Sigma$ and in fact that it agrees on $\Sigma \times D_{0}{ }^{*}$ with the central part of $\bar{\partial}_{\tilde{A}}$. It then follows that $\operatorname{det}\left(g_{0}\right)$ is a holomorphic function from $D_{0}^{*}$ to $\mathbf{C}^{*}$.

Claim 6.4 The winding number of $\operatorname{det}\left(g_{0}\right)$ is even.

Assume for now that the claim is true.

Then we may replace $g_{0}$ by $h=\frac{1}{\sqrt{\operatorname{det} g_{0}}} g_{0}$; this is a section of $S L(E)$ over $\Sigma \times D_{0}{ }^{*}$, that is a complex gauge automorphism whose determinant is the constant function 1 . Since $\frac{1}{\sqrt{\operatorname{det} g_{0}}}$ is a holomorphic function, $h$ also satisfies the equation

$$
\bar{\partial}_{B}=h^{-1} \circ \bar{\partial}_{\tilde{A}} \circ h .
$$

Also as $z \rightarrow 0,\left.h\right|_{\Sigma_{z}}$ must be converging to an element in the stabiliser of $\bar{\partial}_{a_{-\infty}}$. That is to say,

$$
\left.h\right|_{\Sigma_{z}} \rightarrow \pm \mathbf{1} \quad \text { as } z \rightarrow 0 .
$$

(The stabiliser consists of constants times the identity, since stable bundles are simple [9, page 88].) We may suppose it is converging to $+\mathbf{1}$ (multiply $h$ by -1 if necessary).

It now follows that the hermitian metric extends, at least continuously, to the extension over $\Sigma_{0}$ given by $h$. 
To establish Equation (3) we will modify the extended bundle. The fact that $h$ has a continuous extension to $\Sigma \times D_{0}$ with $\left.h\right|_{\Sigma_{0}}=\mathbf{1}$ in fact shows that $h$ is continuously homotopic to the constant identity gauge automorphism. Indeed a homotopy is given by

$$
H_{s}(z)=h(z-s z)
$$

(Using the identification

$$
\left.\Gamma\left(\Sigma \times D_{0}{ }^{*}, S L(E)\right)=\operatorname{Maps}\left(D_{0}{ }^{*}, \Gamma\left(\Sigma, S L\left(E_{\Sigma}\right)\right)\right) .\right)
$$

Thus we may replace $h$ by the identity, at each end, to get a topologically equivalent smooth bundle $\mathcal{E}^{\prime}$ over $\Sigma \times \mathbf{C P}^{\mathbf{1}}$. Now the metric clearly extends to a smooth hermitian metric on this bundle, and we can produce a smooth unitary connection $A^{\prime}$ on $\mathcal{E}^{\prime}$ by "damping down" the original connection $A$ to the limiting connections $\pi_{\Sigma}^{*} a_{ \pm \infty}$ on each end of the cylinder. This is a standard technique.

Start by choosing some $T>>0$. It follows from Theorem 3.3 and our choice of gauge representatives for $[\tilde{A}]$ on the ends of the cylinder that we can write

$$
\tilde{A}=\pi_{\Sigma}^{*} a_{ \pm \infty}+\alpha_{ \pm}
$$

with

$$
\left\|\alpha_{ \pm}\right\|_{L_{2}^{2}\left(Y \times\left[ \pm T-\frac{1}{2}, \pm T+\frac{1}{2}\right]\right)} \leq C e^{-\delta|T|} .
$$

We choose a smooth function $\beta_{+}: \mathbf{R} \rightarrow \mathbf{R}$ with

$$
\beta_{+}(t)=\left\{\begin{array}{lll}
1 & \text { if } & t>T+\frac{1}{2} \\
0 & \text { if } & t<T-\frac{1}{2}
\end{array}\right.
$$

Also define $\beta_{-}(t)=\beta_{+}(-t)$. Now the connection

$$
A^{\prime}=\tilde{A}-\beta_{ \pm} \cdot \alpha_{ \pm}
$$

extends to the bundle $\mathcal{E}^{\prime}$, since it is equal to $\pi_{\Sigma}^{*} a_{ \pm \infty}$ on the ends.

Computing the curvature, we get

$$
\begin{aligned}
F_{A^{\prime}}=F_{\tilde{A}} & -\beta_{+} \cdot d_{\tilde{A}} \alpha_{+}-d \beta_{+} \wedge \alpha_{+}+\beta_{+}{ }^{2} \cdot \alpha_{+} \wedge \alpha_{+} \\
& -\beta_{-} \cdot d_{\tilde{A}} \alpha_{-}-d \beta_{-} \wedge \alpha_{-}+\beta_{-}{ }^{2} \cdot \alpha_{-} \wedge \alpha_{-} .
\end{aligned}
$$

By making $T$ large enough, it follows from (4) and the usual Sobolev multiplication theorems that the integrals $\int_{S} \operatorname{Tr} F_{A^{\prime}}^{0} \wedge F_{A^{\prime}}^{0}$ and $\int_{\Sigma \times S^{1} \times \mathbf{R}} \operatorname{Tr} F_{\tilde{A}}^{0} \wedge F_{\tilde{A}}^{0}=e(A)$ may be made arbitrarily close. Since both are integer multiples of $4 \pi^{2}$, they must in fact be the same, as required. 
Proof of Claim 6.4 The connection $\mathcal{I}(\tilde{A})$ (see Lemma 3.4) is the image of $\tilde{A}$ under a complex gauge transformation whose determinant has winding number 1 around $z=0$. Suppose that the winding number of $\operatorname{det}\left(g_{0}\right)$ is odd. Then we may write

$$
\bar{\partial}_{B}=\left(g_{0}^{\prime}\right)^{-1} \circ \bar{\partial}_{\mathcal{I}(\tilde{A})} \circ g_{0}^{\prime},
$$

with $\operatorname{det}\left(g_{0}^{\prime}\right)$ even. Then as above we may divide by the square root of the determinant to obtain a section $h$ of $S L(E)$ over $\Sigma \times D_{0}^{*}$ satisfying

$$
\bar{\partial}_{B}=(h)^{-1} \circ \bar{\partial}_{\mathcal{I}(\tilde{A})} \circ h .
$$

We will show that in fact this is not possible by comparing the asymptotic limits of the operators $\bar{\partial}_{B}$ and $\bar{\partial}_{\mathcal{I}(\tilde{A})}$ on the end of the cylinder.

Since $\bar{\partial}_{B}$ extends smoothly over $\Sigma \times D_{0}$, and its restriction to $\Sigma_{0}$ is $\bar{\partial}_{a_{-\infty}}$ we have

$$
\left.\lim _{t \rightarrow-\infty} \bar{\partial}_{B}\right|_{\Sigma_{(\theta, t)}}=\bar{\partial}_{a_{-\infty}},
$$

with convergence in $C^{\infty}$ and hence also in $L_{1}^{2}$, uniformly in $\theta$.

Theorem 3.3 and the trace theorem for Sobolev spaces (see for example [3]) imply that

$$
\lim _{t \rightarrow-\infty} \bar{\partial}_{\mathcal{I}(\tilde{A})}\left|\Sigma_{(\theta, t)}=\bar{\partial}_{\mathcal{I}\left(\pi_{\Sigma}^{*} a_{-\infty}\right)}\right| \Sigma_{(\theta, t)},
$$

where the limit is taken in the $L_{1}^{2}$ topology. By the continuity property of the trace operator, this convergence is also uniform in $\theta$. Note that the restriction $\bar{\partial}_{\mathcal{I}\left(\pi_{\Sigma}^{*} a_{-\infty}\right)} \mid \Sigma_{(\theta, t)}$ is independent of $t$; in fact it follows from the definition of $\mathcal{I}$ that

$$
\bar{\partial}_{\mathcal{I}\left(\pi_{\Sigma}^{*} a_{-\infty}\right)} \mid \Sigma_{(\theta, t)}=\left(\begin{array}{cc}
e^{\frac{i \theta}{2}} & 0 \\
0 & e^{-\frac{i \theta}{2}}
\end{array}\right)^{*} \bar{\partial}_{a} .
$$

The above uniform convergences, together with the slice condition (see Section 4) imply that

$$
\left.\lim _{t \rightarrow-\infty} h\right|_{\Sigma_{(\theta, t)}}= \pm\left(\begin{array}{cc}
e^{\frac{i \theta}{2}} & 0 \\
0 & e^{-\frac{i \theta}{2}}
\end{array}\right),
$$

with convergence in $L_{2}^{2}$ and hence in $C^{0}$, uniformly in $\theta$. This is a contradiction: it is not possible to have a smooth (or even continuous) complex gauge transformation $h$ on the cylinder $Y \times S^{1}$ converging uniformly to a discontinuous limit on $Y$. 


\section{$7 \quad$ Injectivity of the function $\Psi$}

Proposition 6.1 defines a function

$$
\Psi: \mathcal{M}_{e} / \mathcal{I} \rightarrow \mathcal{Z}_{(\mathcal{D}, c)} .
$$

In this section we establish that the map $\Psi$ is injective.

Proposition 7.1 $\Psi$ is injective. That is, if $[A],\left[A^{\prime}\right] \in \mathcal{M}_{e}$ determine isomorphic holomorphic structures and both $\left[A_{t}\right]$ and $\left[A_{t}^{\prime}\right]$ converge to limits in $\mathcal{R}_{+}$ as $t \rightarrow \infty$, then $[A]=\left[A^{\prime}\right]$.

(Recall that the involution $\mathcal{I}$ switches the components $\mathcal{R}_{+}, \mathcal{R}_{-}$of projectively flat connections over $Y$; fixing $\left[A_{\infty}\right] \in \mathcal{R}_{+}$chooses between $[A]$ and $\mathcal{I}([A])$.)

The proof of Proposition 7.1 uses the maximum principle applied to a function measuring the distance between two metrics, following Donaldson $[4,6]$. (Much of the proof is taken directly from [6]).

If $H$ and $K$ are Hermitian metrics on $E$ (which may be regarded as isomorphisms from $E$ to the dual bundle $E^{*}$ ) then $\eta=H^{-1} K$ is a section of the bundle of endomorphisms End $E$ which is self-adjoint with respect to either metric.

Definition 7.2 For any two hermitian metrics $H, K$ on $E$ set

$$
\sigma(H, K)=\operatorname{Tr}\left(H^{-1} K\right)+\operatorname{Tr}\left(K^{-1} H\right)-4 \quad \in C^{\infty}\left(\Sigma \times S^{1} \times \mathbf{R}\right) .
$$

This has the property that $\sigma(H, K) \geq 0$ with equality if and only if $H=K$.

Let $H=\langle\cdot, \cdot\rangle$ represent the metric on $E$ which is pulled back from that on $E_{\Sigma}$, and with respect to which $A, A^{\prime}$ are unitary. By hypothesis

$$
\bar{\partial}_{A^{\prime}}=g^{-1} \circ \bar{\partial}_{A} \circ g
$$

for some complex gauge transformation $g$, whose determinant we may require to be identically 1 . Let $K$ be the Hermitian metric on $E$ given by

$$
K=\langle g \cdot, g \cdot\rangle=\left\langle\cdot, g^{*} g \cdot\right\rangle=\langle\cdot, \eta \cdot\rangle .
$$

We will show that in fact $g$ is a unitary gauge transformation by demonstrating that $H=K$.

Let $A_{H}$ be the unique connection determined by the metric $H$ and the holomorphic structure $\bar{\partial}_{A}$, and let $A_{K}$ be the connection determined by $K$ and $\bar{\partial}_{A}$. Then in fact $A_{H}=A$, and $A_{K}=g \circ A^{\prime} \circ g^{-1}$. Thus $A_{H}$ is a $U(2)$-instanton, 
while $A_{K}$ is a finite-energy, projectively ASD connection with the same central part as $A_{H}$, but it is unitary with respect to the metric $K$ rather than $H$.

The curvature of these connections is related by

$$
F_{K}=F_{H}+\bar{\partial}_{H}\left(\eta^{-1} \partial_{H} \eta\right) .
$$

For an integrable connection $A$ in a vector bundle over a compact Kähler surface, it is not hard to show that the condition that $A$ is a $U(2)$-instanton (ie $\operatorname{Tr} F_{A}$ harmonic, $F_{A}^{0} \mathrm{ASD}$ ) is equivalent to the Hermitian-Einstein condition

$$
\hat{F_{A}}=\lambda \cdot \mathbf{1}
$$

for constant scalar $\lambda$. (Here $\hat{F_{A}}=\Lambda F_{A}$, where $\Lambda$ is the adjoint of wedging with the Kähler form, and $\mathbf{1}$ is the identity endomorphism of the vector bundle in question).

Generalising to a possibly noncompact Kähler surface, one finds the equivalence

$$
\left\{\begin{array}{cc}
F_{A}^{0} & \text { ASD } \\
\operatorname{Tr} F_{A} & \text { harmonic }
\end{array}\right\} \Leftrightarrow \hat{F}_{A}=f \cdot \mathbf{1},
$$

where $f$ is a harmonic function. In our definition of $U(2)$-instantons on $\Sigma \times$ $S^{1} \times \mathbf{R}$, we require that $\operatorname{Tr} F_{A}$ is both harmonic and pulled back from $\Sigma$. This means that $A_{H}$ and $A_{K}$ both satisfy the Hermitian-Einstein condition $\hat{F}_{A}=\lambda \cdot \mathbf{1}$, with $\lambda$ a fixed constant depending on the first Chern class of the restriction of the bundle to $\Sigma \times\{$ point $\}$.

Lemma $7.3 \Delta \sigma(H, K) \leq 0$.

Proof Applying $i \Lambda$ to (5) and taking the trace yields

$$
\begin{aligned}
0 & =\operatorname{Tr} i \Lambda \bar{\partial}_{H}\left(\eta^{-1} \partial_{H} \eta\right) \\
& =\operatorname{Tr} i \Lambda\left(\eta^{-1} \bar{\partial}_{H} \partial_{H} \eta+\eta^{-1} \bar{\partial}_{H} \eta \eta^{-1} \wedge \partial_{H} \eta\right) .
\end{aligned}
$$

Now we use the Weitzenböck formula (see [7, page 212]): for any connection $A$ on $E$,

$$
\partial_{A}^{*} \partial_{A}=\frac{1}{2} \nabla_{A}^{*} \nabla_{A}-i \hat{F}_{A}
$$

on $\Omega^{0}($ End $E)$, with $i \hat{F}_{A}$ acting by the adjoint action. Here $\nabla_{A}=\partial_{A}+\bar{\partial}_{A}$ is the covariant derivative on End $E$ associated to $A$. We also have the following first order Kähler identity on $\Omega^{1,0}($ End $E)$ :

$$
\partial_{A}^{*}=-i \Lambda \bar{\partial}_{A} .
$$


Applying these identities as well as the Hermitian-Einstein condition to (6) yields

$$
\Delta \operatorname{Tr} \eta=\operatorname{Tr} \nabla_{H}^{*} \nabla_{H} \eta=2 i \Lambda \operatorname{Tr}\left(\bar{\partial}_{H} \eta \eta^{-1} \wedge \partial_{H} \eta\right) .
$$

Choose a frame for $E$ over any given point $p$ which is unitary with respect to $H$ and in which $\eta$ is diagonal with eigenvalues $\lambda_{a}$, and let $\pi_{a b}$ be the matrix entries of $\bar{\partial}_{H} \eta$ at $p$. Then $\partial_{H} \eta$ has entries $\bar{\pi}_{b a}$ and

$$
i \Lambda \operatorname{Tr}\left(\bar{\partial}_{H} \eta \eta^{-1} \wedge \partial_{H} \eta\right)=i \Lambda \sum_{a, b} \lambda_{a}^{-1} \pi_{a b} \wedge \bar{\pi}_{a b}=-\sum_{a, b} \lambda_{a}^{-1}\left|\pi_{a b}\right|^{2} \leq 0 .
$$

(Using the fact that for a $(1,0)$ form $\phi, i \Lambda(\phi \wedge \bar{\phi})=-|\phi|^{2}$.)

Thus $\Delta \operatorname{Tr}\left(H^{-1} K\right) \leq 0$. Interchanging $H, K$ we see that $\operatorname{Tr}\left(K^{-1} H\right)$ is likewise sub-harmonic, and hence also $\sigma(H, K)$.

The maximum principle (see for example [11]) states that a subharmonic function on a compact domain attains its maximum value on the boundary. In order to apply this we need to see what happens to $\sigma(H, K)$ on the ends of the cylinder $\Sigma \times S^{1} \times \mathbf{R}$.

Lemma 7.4 $\sigma(H, K) \rightarrow 0 \quad$ as $\quad t \rightarrow \pm \infty$.

Proof Consider again the $U(2)$-instantons $A, A^{\prime}$ which are related by the complex gauge transformation $g$ with determinant 1 . By Theorem 3.2, both $\left[A_{t}\right],\left[A_{t}^{\prime}\right]$ converge to flat limits as $t \rightarrow \pm \infty$. Using Theorem 3.3 and the Sobolev trace theorem $([3]),\left.A\right|_{\Sigma_{(\theta, t)}}$ and $\left.A^{\prime}\right|_{\Sigma_{(\theta, t)}}$ converge in $L_{1}^{2}$ to projectively flat limits on $\Sigma$ as $t \rightarrow \pm \infty$, uniformly in $\theta$. These flat limits determine isomorphic holomorphic structures, so by the Narasimhan-Seshadri theorem they are in the same unitary gauge orbit. Arguing as in the proof of Claim 6.4 (see also proof of Lemma 8.1) we find that $\left.g\right|_{\Sigma_{(\theta, t)}}$ is converging in $C^{0}$ to a unitary limit for each $\theta$ as $t \rightarrow \pm \infty$, uniformly in $\theta$. Thus $\eta=g^{*} g$ and $\eta^{-1}$ are converging to the identity in $C^{0}$ as $t \rightarrow \pm \infty$, from which it follows that $\sigma(H, K) \rightarrow 0$.

Proof of Proposition 7.1. Let $H, K$ be the metrics obtained from $A, A^{\prime}$ as above. For any $\epsilon>0$, it follows from Lemma 7.4 that we may choose $T>0$ such that $\sigma(H, K)<\epsilon$ for $|t| \geq T$. Lemma 7.3 tells us that $\Delta \sigma(H, K) \leq 0$. It follows from the maximum principle that $\sigma(H, K)<\epsilon$ on $\Sigma \times S^{1} \times[-T, T]$ and thus on all of $\Sigma \times S^{1} \times \mathbf{R}$. Thus $\sigma(H, K)=0$ at each point, and $H=K$. It follows that the gauge transformation $g$ is unitary, so that

$$
[A]=\left[A^{\prime}\right]
$$

in $\mathcal{M}_{e}$, as required. 


\section{Some convergence results}

In this section we prove some convergence results for complex gauge transformations. These will be used to prove that $\Psi$ is surjective and that $\Psi$ and its inverse are continuous.

Throughout this section $E_{\Sigma} \rightarrow \Sigma$ is a smooth rank two hermitian vector bundle with degree one, and for any subset $W \subset \mathbf{C P}^{1}$, we denote by

$$
E \rightarrow \Sigma \times W
$$

the pullback of $E_{\Sigma}$ by the projection onto the first factor. The term $\bar{\partial}$-operator refers to smooth $(0,1)$ connections.

In the following two lemmas, take $W$ to be an annulus in $\mathbf{C P}^{\mathbf{1}}$. Any time we refer to an annulus contained in another annulus, it is to be understood that the larger annulus retracts onto the smaller one.

Lemma 8.1 Suppose $\left\{\bar{\partial}_{A_{n}}\right\}_{n=1}^{\infty},\left\{\bar{\partial}_{B_{n}}\right\}_{n=1}^{\infty}, \bar{\partial}_{A}$ and $\bar{\partial}_{B}$ are $\bar{\partial}$-operators on $E \rightarrow \Sigma \times W$ whose restrictions to $\Sigma \times\{z\}$ for each $z \in W$ are stable with fixed determinant $\mathcal{L}$, and that

$$
\begin{aligned}
& \bar{\partial}_{A_{n}} \rightarrow \bar{\partial}_{A}, \\
& \bar{\partial}_{B_{n}} \rightarrow \bar{\partial}_{B}
\end{aligned}
$$

in $C^{\infty}$ on compact subsets of $\Sigma \times W$. Suppose also that

$$
\begin{aligned}
\bar{\partial}_{B_{n}} & =\phi_{n}^{*} \bar{\partial}_{A_{n}}, \\
\bar{\partial}_{B} & =\phi^{*} \bar{\partial}_{A}
\end{aligned}
$$

where $\left\{\phi_{n}\right\}_{n=1}^{\infty}, \phi$ are sections of $S L(E)$ over $\Sigma \times W$. Then $\left\{\phi_{n}\right\}$ is uniformly bounded in $C^{0}\left(\Sigma \times W_{0}\right)$, where $W_{0}$ is a compact annulus contained in $W$.

Proof Recall from Section 4 that there are local slices for the action of the complex gauge group on the space of $(0,1)$ connections over $\Sigma$.

For each $z \in W$, let $\bar{\partial}_{z}$ denote the $(0,1)$ connection on $E_{\Sigma} \rightarrow \Sigma$ given by the restriction of $\bar{\partial}_{A}$ to $\Sigma \times\{z\}$. Denote by

$$
S_{z}=\bar{\partial}_{z}+\operatorname{Ker} \bar{\partial}_{z}^{*}
$$

the slice in $\mathcal{A}_{0}^{(0,1)}$ through $\bar{\partial}_{z}$. Let $U_{z}$ denote the neighbourhood of $\bar{\partial}_{z}$ in the slice with the property that the natural map

$$
U_{z} \times{ }_{ \pm 1} \mathcal{G}_{0}^{c} \rightarrow \mathcal{A}_{0}^{(0,1)}
$$


is a diffeomorphism onto its image. By continuity of $\bar{\partial}_{A}$ and by the $C^{\infty}$ convergence $\bar{\partial}_{A_{n}} \rightarrow \bar{\partial}_{A}$, it follows that for some large $N$ and some open disk neighbourhood $D_{z}$ of $z$ in $W$, the restriction of $\bar{\partial}_{A_{n}}$ to $\Sigma \times\left\{z^{\prime}\right\}$ is in the image of $U_{z} \times_{ \pm 1} \mathcal{G}_{0}$ for all $n \geq N$ and for all $z^{\prime} \in D_{z}$. Then since

$$
\bar{\partial}_{A_{n}} \rightarrow \bar{\partial}_{A}
$$

and

$$
\phi_{n}^{*} \bar{\partial}_{A_{n}} \rightarrow \phi^{*} \bar{\partial}_{A}
$$

it follows that for any neighbourhood $O$ of the identity in $\mathcal{G}_{0}^{c}$, there exists some $N^{\prime}$ such that $\left.\left( \pm \phi^{-1} \phi_{n}\right)\right|_{\Sigma \times\left\{z^{\prime}\right\}} \in O$ for all $n \geq N^{\prime}$ and for all $z^{\prime} \in D_{z}$.

It follows that the $C^{0}$ norms of $\phi^{-1} \phi_{n}$ and hence also of $\phi_{n}$ are uniformly bounded on $\Sigma \times D_{z}$. Choosing a finite cover of $\Sigma \times W_{0}$ by such sets $\Sigma \times D_{z}$ then yields the desired result.

Lemma 8.2 Suppose $\left\{\bar{\partial}_{A_{n}}\right\}_{n=1}^{\infty},\left\{\bar{\partial}_{B_{n}}\right\}_{n=1}^{\infty}, \bar{\partial}_{A}$ and $\bar{\partial}_{B}$ are $\bar{\partial}$-operators on $E \rightarrow \Sigma \times W$, and that

$$
\begin{aligned}
& \bar{\partial}_{A_{n}} \rightarrow \bar{\partial}_{A} \\
& \bar{\partial}_{B_{n}} \rightarrow \bar{\partial}_{B}
\end{aligned}
$$

in $C^{\infty}\left(\Sigma \times W_{0}\right)$, where $W_{0}$ is a compact annulus contained in $W$. Suppose also that

$$
\bar{\partial}_{B_{n}}=\phi_{n}^{*} \bar{\partial}_{A_{n}},
$$

where $\left\{\phi_{n}\right\}$ are a sequence of sections of $S L(E)$ over $\Sigma \times W$ which are uniformly bounded in $C^{0}\left(\Sigma \times W_{0}\right)$. Then there exists a subsequence of $\left\{\phi_{n}\right\}$ which is converging to some limit $\phi$ in $C^{\infty}\left(\Sigma \times W_{\infty}\right)$ with

$$
\bar{\partial}_{B}=\phi^{*} \bar{\partial}_{A},
$$

where $W_{\infty} \subset W_{0}$ is a slightly smaller compact annulus.

Proof This is a standard bootstrap argument.

The above lemmas will enable us to prove a very useful convergence result. We will first describe the rather complicated hypotheses.

Recall from Section 4 that $\mathcal{U}$ is the universal bundle over $\Sigma \times \mathcal{N}(\Sigma)$.

Let $U$ and $V$ be two possibly disconnected subsets of $\mathbf{C P}^{1}$ such that

$$
\mathbf{C P}^{1}=U \cup V,
$$

and $W=U \cap V$ is a disjoint union of finitely many open annuli. Let $X$ be any subset of $\Sigma \times(U-W)$. Let $\left\{\bar{\partial}_{A_{n}}\right\}_{n=1}^{\infty}, \bar{\partial}_{A}$ be $\bar{\partial}$-operators on $E \rightarrow \Sigma \times U$, with the following properties: 
- $\bar{\partial}_{A_{n}} \rightarrow \bar{\partial}_{A}$ in $C^{\infty}$ on compact subsets of $(\Sigma \times U)-X$,

- $\left(E, \bar{\partial}_{A_{n}}\right) \cong\left(\operatorname{Id} \times f_{n}\right)^{*} \mathcal{U}$ on $\Sigma \times W$, and

- $\left(E, \bar{\partial}_{A}\right) \cong\left(\operatorname{Id} \times f_{\infty}\right)^{*} \mathcal{U}$ on $\Sigma \times W$,

where $f_{n}: \mathbf{C P}^{\mathbf{1}} \rightarrow \mathcal{N}(\Sigma)$ are a sequence of holomorphic maps converging in $C^{\infty}$ on compact subsets of $\Sigma \times V$ to a limit $f_{\infty}$.

One may form bundles $\mathcal{E}_{n}$ over $\Sigma \times \mathbf{C P}^{\mathbf{1}}$ by gluing $\left(E, \bar{\partial}_{A_{n}}\right)$ and $\left.\left(\operatorname{Id} \times f_{n}\right)^{*} \mathcal{U}\right|_{\Sigma \times V}$ along the overlap region $W$, and similarly a bundle $\mathcal{E}_{\infty}$ from $\left(E, \bar{\partial}_{A}\right)$ and $\left.\left(\operatorname{Id} \times f_{\infty}\right)^{*} \mathcal{U}\right|_{\Sigma \times V}$

Corollary 8.3 Let $\left[\mathcal{E}_{n}\right]$ and $\left[\mathcal{E}_{\infty}\right]$ be as described above. If all of $\left[\mathcal{E}_{n}\right]$ have isomorphic determinant line bundles and the same second Chern class, then each $\bar{\partial}_{A_{n}}$ may be extended to a $\bar{\partial}$-operator $\bar{\partial}_{n}$ on a smooth vector bundle $\tilde{E} \rightarrow S$, and $\bar{\partial}_{A}$ may be extended to $\bar{\partial}_{\infty}$ on $\left.\tilde{E}\right|_{S-X}$, so that the following hold:

- $\left(\tilde{E}, \bar{\partial}_{n}\right) \cong \mathcal{E}_{n}$,

- after passing to a subsequence, $\bar{\partial}_{n}$ converge to a limit $\bar{\partial}_{\infty}$ in $C^{\infty}$ on compact subsets of $S-X$, and

- $\left.\left.\left(\tilde{E}, \bar{\partial}_{\infty}\right)\right|_{S-X} \cong \mathcal{E}_{\infty}\right|_{S-X}$.

In particular if $X=\emptyset$, then (after passing to a subsequence) $\left[\mathcal{E}_{n}\right] \rightarrow\left[\mathcal{E}_{\infty}\right]$ in $\mathcal{M}_{(\mathcal{D}, c)}$.

Proof Denote by $E_{V} \rightarrow \Sigma \times V$ the pullback of $E_{\Sigma} \rightarrow \Sigma$. For each $n$ we may choose $\bar{\partial}_{V_{n}}$ on $E_{V}$ so that

$$
\left.\left(E_{V}, \bar{\partial}_{V_{n}}\right) \cong\left(\operatorname{Id} \times f_{n}\right)^{*} \mathcal{U}\right|_{\Sigma \times V}
$$

These may be chosen so that $\lim _{n \rightarrow \infty} \bar{\partial}_{V_{n}}=\bar{\partial}_{V}$, in $C^{\infty}$ on compact subsets of $\Sigma \times V$, where

$$
\left.\left(E_{V}, \bar{\partial}_{V}\right) \cong\left(\operatorname{Id} \times f_{\infty}\right)^{*} \mathcal{U}\right|_{\Sigma \times V} .
$$

We may also require that the central part of each $\bar{\partial}_{V_{n}}$ is pulled back from $\Sigma$, and is the same for each $n$. Identifying the bundles $E$ and $E_{V}$ over $\Sigma \times W$ in the obvious way, it follows that

$$
\begin{aligned}
\bar{\partial}_{V_{n}} & =\phi_{n}^{*} \bar{\partial}_{A_{n}}, \\
\bar{\partial}_{V_{\infty}} & =\phi^{*} \bar{\partial}_{A}
\end{aligned}
$$

for some sections $\phi_{n}, \phi$ of $G L(E)$ over $\Sigma \times W$. Replace the central parts of $\bar{\partial}_{A_{n}}$ and $\bar{\partial}_{A}$ by the same form pulled back from $\Sigma$; it then follows that the determinants of $\phi_{n}$ and $\phi$ will be holomorphic functions from $W$ to $\mathbf{C}^{*}$. For 
each $n$, the function det $\phi_{n}$ will have either odd or even winding number around each component annulus in $W$. Pass to a subsequence for which the parities of these winding numbers are the same for all $n$.

On an annulus on which the winding number is even, $\phi_{n}$ may be replaced by $\phi_{n}^{\prime}=\frac{1}{\sqrt{\operatorname{det} \phi_{n}}} \phi_{n}$. This is a section of $S L(E)$ over this annulus, satisfying

$$
\bar{\partial}_{V_{n}}=\phi_{n}^{* *} \bar{\partial}_{A_{n}} .
$$

It follows from the previous two lemmas that (after passing to a subsequence) these gauge automorphisms converge to a limit $\phi^{\prime}$ which satisfies

$$
\bar{\partial}_{V}=\phi^{*} \bar{\partial}_{A} .
$$

On an annulus on which the winding number is odd, choose a holomorphic function $\alpha$ from the annulus to $\mathbf{C}^{*}$ with winding number 1 , and let $\psi=\left(\begin{array}{ll}\alpha & 0 \\ 0 & 1\end{array}\right)$. Then $\psi$ is a section of $G L(E)$ over $\Sigma \times W$. The determinant of $\psi \cdot \phi_{n}$ is even for all $n$, and

$$
\bar{\partial}_{V_{n}}=\left(\psi \cdot \phi_{n}\right)^{*}\left(\psi^{-1}\right)^{*} \bar{\partial}_{A_{n}} .
$$

But then the previous two lemmas apply to the sequence of $S L$-automorphisms

$$
\frac{1}{\sqrt{\operatorname{det} \psi \cdot \phi_{n}}} \psi \cdot \phi_{n}
$$

It follows that $\phi_{n}^{\prime}=\frac{1}{\sqrt{\operatorname{det} \psi \cdot \phi_{n}}} \phi_{n}$ satisfy

$$
\bar{\partial}_{V_{n}}=\phi_{n}^{* *} \bar{\partial}_{A_{n}}
$$

on this annulus, and also that the sequence $\phi_{n}^{\prime}$ has a convergent subsequence.

Summing up then, we see that we can find a sequence $\phi_{n}$ of sections of $G L(E)$ over $\Sigma \times W$ which satisfy

$$
\bar{\partial}_{V_{n}}=\phi_{n}^{*} \bar{\partial}_{A_{n}}
$$

on $\Sigma \times W$, and which have a subsequence which is converging in $C^{\infty}$ on compact subsets of $\Sigma \times W$ to a limit $\phi$ which satisfies

$$
\bar{\partial}_{V}=\phi^{*} \bar{\partial}_{A} \text {. }
$$

The required operators $\bar{\partial}_{n}$ are then given by gluing $\bar{\partial}_{A_{n}}$ and $\bar{\partial}_{V_{n}}$ along $\Sigma \times W$ by the automorphisms $\phi_{n}$.

We may describe them in a way that shows clearly that they are extensions of $\bar{\partial}_{A_{n}}$ on a fixed bundle $\tilde{E}$. We know that $\phi_{n}^{-1} \phi_{N}$ are converging to the identity in $C^{\infty}$ on compact subsets of $\Sigma \times W$. Choose $N$ large enough so that $\phi_{n}^{-1} \phi_{N}$ 
is homotopic to the identity for all $n \geq N$. Define $\tilde{E}$ to be the bundle formed from $E \rightarrow \Sigma \times U$ and $E_{V} \rightarrow \Sigma \times V$ using the transition function $\phi_{N}$ on $\Sigma \times W$. Then

$$
\begin{aligned}
\bar{\partial}_{n} & =\bar{\partial}_{A_{n}} \cup_{\phi_{n}} \bar{\partial}_{V_{n}} \\
& =\bar{\partial}_{A_{n}} \cup_{\phi_{N}}\left(\phi_{n}^{-1} \phi_{N}\right)^{*} \bar{\partial}_{V_{n}},
\end{aligned}
$$

for some extension of $\phi_{n}^{-1} \phi_{N}$ over $\Sigma \times V$, chosen so that $\phi_{n}^{-1} \phi_{N}$ converge to the identity on compact subsets of $\Sigma \times V$.

We can now prove that the two topologies on $\mathcal{M}_{(\mathcal{D}, c)}$ given in Section 5 agree.

Proposition 8.4 The correspondence given in Proposition 5.2 between $\mathcal{M}_{(\mathcal{D}, c)}$ and $\mathcal{M}_{(\mathcal{D}, c)}^{\prime}$ is a homeomorphism. That is to say, a sequence $\left[\mathcal{E}_{n}\right]$ converges to $[\mathcal{E}]$ in $\mathcal{M}_{(\mathcal{D}, c)}$ if and only if the corresponding sequence of triples $\left(f_{n}, \cup_{i=1}^{k_{n}} z_{i ; n}, \cup_{i=1}^{k_{n}}\left[\mathcal{E}_{i ; n}\right]\right)$ converges to the triple $\left(f_{\mathcal{E}}, \cup_{i=1}^{k} z_{i}, \cup_{i=1}^{k}\left[\mathcal{E}_{i}\right]\right)$ associated to $[\mathcal{E}]$ in the following way:

- $f_{n} \rightarrow f_{\mathcal{E}}$ in $C^{\infty}$ on compact subsets of $\mathbf{C P}^{\mathbf{1}}-\cup_{i=1}^{k} z_{i}$

- for any open neighbourhood $D$ of $\cup_{i=1}^{k} z_{i}$, there exists $N$ such that $\cup_{i=1}^{k_{n}} z_{i ; n} \subset D \quad \forall \quad n \geq N$

- for some open neighbourhood $D$ of $\cup_{i=1}^{k} z_{i}$, the bundles determined by $f_{n}$ and $\cup_{i=1}^{k_{n}}\left[\mathcal{E}_{i ; n}\right]$ converge in $C^{\infty}$ on $\Sigma \times D$ to that determined by $f_{\mathcal{E}}$ and $\cup_{i=1}^{k}\left[\mathcal{E}_{i}\right]$.

Proof Suppose first that $\left[\mathcal{E}_{n}\right]$ converges to $[\mathcal{E}]$ in $\mathcal{M}_{(\mathcal{D}, c)}$. We must show that the associated sequence of triples satisfies the 3 conditions above. The third requirement is satisfied immediately by taking $D=\mathbf{C P}^{\mathbf{1}}$. Let us consider the second. Let $D$ be any open set in $\mathbf{C P}^{\mathbf{1}}$ containing $\cup_{i=1}^{k} z_{i}$ and suppose that after passing to a subsequence at least one of the points $\cup_{i=1}^{k_{n}} z_{i ; n}$ is in the complement of $D$ for all $n$. Then a subsequence $z_{i_{n} ; n}$ is converging to a limit point $z$ in the compact set $\mathbf{C P}^{\mathbf{1}}-D$. By continuity and the fact that stability is an open condition, it follows that the limiting bundle $[\mathcal{E}]$ must have unstable restriction to $\Sigma \times\{z\}$, which is a contradiction.

It now follows that for any neighbourhood $D$ of $\cup_{i=1}^{k} z_{i}$ in $\mathbf{C P}^{\mathbf{1}}$ the restriction of $\mathcal{E}_{n}$ to $\Sigma \times\left(\mathbf{C P}^{\mathbf{1}}-D\right)$ is stable on every $\Sigma \times\{z\}$, for large $n$. Thus the maps $f_{n}$ are converging to $f_{\mathcal{E}}$ in $C^{\infty}$ on $\mathbf{C P}^{\mathbf{1}}-D$, and hence in $C^{\infty}$ on compact subsets of $\mathbf{C P}^{\mathbf{1}}-\cup_{i=1}^{k} z_{i}$.

Conversely, suppose $\left(f_{n}, \cup_{i=1}^{k_{n}} z_{i ; n}, \cup_{i=1}^{k_{n}}\left[\mathcal{E}_{i ; n}\right]\right)$ converges to $\left(f_{\mathcal{E}}, \cup_{i=1}^{k} z_{i}, \cup_{i=1}^{k}\left[\mathcal{E}_{i}\right]\right)$. Let $U$ be a disjoint union of open disks of radius $r$ centred at each of $\cup_{i=1}^{k} z_{i}$, 
and let $U_{\frac{1}{2}} \subset U$ be closed disks of radius $r / 2$, also centred at $\cup_{i=1}^{k} z_{i}$. Choose $r$ small enough so that the restriction of $\left[\mathcal{E}_{n}\right]$ is converging to the restriction of $[\mathcal{E}]$ on $U$ (which we can do by the third condition in the definition of convergence of triples). By the second condition above, there exists $N$ such that $\cup_{i=1}^{k_{n}} z_{i ; n} \subset U_{\frac{1}{2}}^{\circ}$ for all $n \geq N$.

Let $V=\mathbf{C P}^{\mathbf{1}}-U_{\frac{1}{2}}$. Then $\mathbf{C P}^{\mathbf{1}}=U \cup V$, and $W=U \cap V$ is a union of open annuli. We may choose $\bar{\partial}$-operators $\left\{\bar{\partial}_{A_{n}}\right\}_{n=1}^{\infty}, \bar{\partial}_{A}$ on $E \rightarrow \Sigma \times U$ so that

$$
\left(E, \bar{\partial}_{A_{n}}\right) \cong \mathcal{E}_{n},\left(E, \bar{\partial}_{A}\right) \cong \mathcal{E}
$$

on $\Sigma \times U$, and

$$
\bar{\partial}_{A_{n}} \rightarrow \bar{\partial}_{A}
$$

in $C^{\infty}$ on compact subsets of $\Sigma \times U$.

It follows from Corollary 8.3 that, after passing to a subsequence,

$$
\left[\mathcal{E}_{n}\right] \rightarrow[\mathcal{E}] .
$$

In fact the same argument shows that any subsequence of $\left\{\left[\mathcal{E}_{n}\right]\right\}$ has a subsequence converging to $[\mathcal{E}]$. Then since $\mathcal{M}_{(\mathcal{D}, \sigma)}$ has a Hausdorff compactification, it follows that (for the entire sequence)

$$
\left[\mathcal{E}_{n}\right] \rightarrow[\mathcal{E}]
$$

as required.

The following lemma is based on arguments used by Donaldson [4] and Morgan [13], and in fact the proof closely follows that in [13, Section 4.2].

Lemma 8.5 Let $X=\cup_{i=1}^{l} x_{i}$ be a finite set of points in $S$, and let $\tilde{E} \rightarrow S$ be a smooth rank 2 hermitian bundle. Suppose that $B_{n}$ are a sequence of unitary connections on $\tilde{E}$ with the following properties:

- $B_{n}$ converge to $B_{\infty}$ in $C^{\infty}$ on compact subsets of $S-X$, where $B_{\infty}$ is a unitary connection on $\left.\tilde{E}\right|_{S-X}$;

- each $B_{n}$ is projectively $A S D$ in some fixed neighbourhood $O$ of $X$ (with respect to some fixed chosen Kähler metric on $S$ ).

Suppose each $\left(\tilde{E}, \bar{\partial}_{B_{n}}\right)$ represents an element of $\mathcal{M}_{(\mathcal{D}, c)}$, and $\left[\left(\tilde{E}, \bar{\partial}_{B_{n}}\right)\right]$ converge to a limit $[\xi]$ in $\mathcal{M}_{(\mathcal{D}, c)}$. Suppose also that

$$
\left.\left(\left.\tilde{E}\right|_{S-X}, \bar{\partial}_{B \infty}\right) \cong \zeta\right|_{S-X}
$$

for some $\mathcal{C}_{\sigma}$-stable bundle $\zeta \rightarrow S$. Then $\xi \cong \zeta$. 
Proof The key point, as in [4] and [13], is to get uniform bounds on $\left|\hat{F}_{B_{n}}\right|$. In both of the references just cited, these bounds follow from the fact that the connections in question are ASD. In fact though since the sequence of connections are converging uniformly on compact subsets of $S-X$, it is sufficient that they be ASD near $X$.

First change the connections $B_{n}$ and $B_{\infty}$ in the following way. Give them all the same fixed central part. Since they all determine isomorphic holomorphic structures on the determinant line bundle this will not affect the convergence on $S-X$ or the isomorphism classes of the holomorphic structures they determine on $\tilde{E}$.

Since $\left[\left(\tilde{E}, \bar{\partial}_{B_{n}}\right)\right] \rightarrow[\xi]$, it follows that there exist $\bar{\partial}$-operators $\bar{\partial}_{n}^{\prime}, \bar{\partial}_{\infty}^{\prime}$ on $\tilde{E}$ with $\lim _{n \rightarrow \infty} \bar{\partial}_{n}^{\prime}=\bar{\partial}_{\infty}^{\prime},\left(\tilde{E}, \bar{\partial}_{\infty}^{\prime}\right) \cong \xi$, and

$$
j_{n} \circ \bar{\partial}_{B_{n}}=\bar{\partial}_{n}^{\prime} \circ j_{n}
$$

for some complex linear automorphism $j_{n}$ of $\tilde{E}$. Let $B_{n}^{\prime}$ denote the unique unitary connection on $\tilde{E}$ which is compatible with the complex structure $\bar{\partial}_{n}^{\prime}$ for each $n$.

Claim 8.6 $\left|\hat{F}_{B_{n}}\right|$ and $\left|\hat{F}_{B_{n}^{\prime}}\right|$ are bounded over $S$ uniformly in $n$.

Proof $\left|\hat{F}_{B_{n}^{\prime}}\right|$ are uniformly bounded over $S$ since $\bar{\partial}_{n}^{\prime}$ are converging uniformly to $\bar{\partial}_{\infty}^{\prime}$, and so $B_{n}^{\prime}$ are also converging uniformly.

Similarly $\left|\hat{F}_{B_{n}}\right|$ are uniformly bounded on compact subsets of $S-X$ since $B_{n}$ is converging uniformly on $S-X$ to $B_{\infty} \cdot\left|\hat{F}_{B_{n}}\right|$ are uniformly bounded over $O$ since the $B_{n}$ are projectively ASD on $O$ with a fixed central part.

Thus $\left|\hat{F}_{B_{n}}\right|$ are uniformly bounded over all of $S$.

Set $\tau_{n}=\operatorname{Tr}\left(j_{n}^{*} \circ j_{n}\right)$, where $j_{n}^{*}$ is the adjoint of $j_{n}$ with respect to the metric on $\tilde{E}$.

Claim 8.7 There exists a constant $C>0$ such that for all $n$

$$
\sup _{x \in S} \tau_{n}(x) \leq C\left\|\tau_{n}\right\|_{L^{2}(S)} .
$$

Proof This follows from the previous claim. (See [13, Corollary 4.2.9].) 
Multiply each $j_{n}$ by a constant $\lambda_{n}$ so that for all $n$

$$
\left\|j_{n}\right\|_{L^{4}(S)}=\left\|\tau_{n}\right\|_{L^{2}(S)}=1 .
$$

Then for all $n$ we have that

$$
\sup _{x \in S} \tau_{n}(x) \leq C .
$$

Now choose balls $B_{i}$ centred at each point $x_{i} \in X$ sufficiently small so that they are disjoint from one another and so that the bundles $\xi$ and $\zeta$ are holomorphically trivial over each $B_{i}$. Let $B=\cup_{i=1}^{l} B_{i}$ and let $T=S-B$. The balls $B_{i}$ should also be chosen so that the volume of $B$ is at most $\frac{1}{2 C}$ (so that $\left.\left\|j_{n}\right\|_{L^{4}(B)} \leq \frac{1}{2}\right)$.

Write

$$
\begin{aligned}
\bar{\partial}_{n}^{\prime} & =\bar{\partial}_{\infty}^{\prime}+\alpha_{n} \\
\bar{\partial}_{B_{n}} & =\bar{\partial}_{B_{\infty}}+\beta_{n}
\end{aligned}
$$

where $\alpha_{n}, \beta_{n}$ converge to 0 in $C^{\infty}$ on $T$. Then (7) becomes

$$
\bar{\partial}_{\infty}^{\prime} \circ j_{n}-j_{n} \circ \bar{\partial}_{B_{\infty}}=j_{n} \beta_{n}-\alpha_{n} j_{n}
$$

Since the $\left\|j_{n}\right\|_{L^{4}(T)}$ are uniformly bounded for all $n$, it follows from this equation and a standard bootstrap argument that there is a subsequence of the $j_{n}$ which converge in $C^{\infty}(T)$ to a limit $j_{\infty}$ which satisfies

$$
\bar{\partial}_{\infty}^{\prime} \circ j_{\infty}-j_{\infty} \circ \bar{\partial}_{B_{\infty}}=0 \text {. }
$$

That is to say $j_{\infty}$ defines a holomorphic map from $\left.\zeta\right|_{T}$ to $\left.\xi\right|_{T}$. Since $\left\|j_{n}\right\|_{L^{4}(T)} \geq$ $\frac{1}{2}$, it follows that $j_{\infty}$ is not the zero map. By making $B$ arbitrarily small, $j_{\infty}$ can be extended to $S-X$. Then by Hartogs' theorem $j_{\infty}$ extends to a nonzero holomorphic map from $\zeta$ to $\xi$. Since these are stable bundles with the same slope, this must in fact be an isomorphism.

The following result is based on Corollary 8.3 and Lemma 8.5 and will be used to prove surjectivity of $\Psi$ and continuity of $\Psi^{-1}$.

Lemma 8.8 Let $\left\{\left[A_{n}\right]\right\}$ be gauge equivalence classes of smooth connections on $E \rightarrow \Sigma \times S^{1} \times \mathbf{R}$ which are converging in $C^{\infty}$ on compact subsets on the complement of some finite set of points to some $[A] \in \mathcal{M}$. Suppose that for any compact cylinder $U \subset S^{1} \times \mathbf{R}$ there exists $N_{U}$ such that $\left.A_{n}\right|_{\Sigma \times U}$ is unitary and projectively $A S D$ for all $n>N_{U}$. Let $\left\{\left[\xi_{n}\right]\right\}$, $[\xi]$ be elements of $\mathcal{Z}_{(\mathcal{D}, c)}$ with $\left[\xi_{n}\right] \rightarrow[\xi]$ as $n \rightarrow \infty$. Suppose also that

$$
\left.\xi_{n}\right|_{\Sigma \times S^{1} \times \mathbf{R}} \cong\left(E, \bar{\partial}_{A_{n}}\right)
$$

as holomorphic bundles. Then $[\xi]=\Psi([A])$. 
Proof Start by decomposing $\mathbf{C P}^{\mathbf{1}}$ into disjoint sets $U$ and $V$. Let $x_{1}, \ldots, x_{l}$ be the "bubble points" in the weak convergence of $\left[A_{n}\right]$ to $[A]$. Choose $V$ to be a sufficiently small neighbourhood of $\{0, \infty\}$ so that

- for some $N, \xi_{n}$ has stable restriction to $\Sigma \times\{z\}$ whenever $n \geq N$ and $z \in V$. (Such a $V$ must exist since $\left[\xi_{n}\right]$ are converging to a limit in $\left.\mathcal{Z}_{(\mathcal{D}, c)} \cdot\right)$

- the points $x_{1}, \ldots, x_{l}$ are all contained in $\Sigma \times\left(\mathbf{C P}^{\mathbf{1}}-V\right)$.

Let $U$ be a cylinder chosen so that $\mathbf{C P}^{1}=U \cup V$ and $W=U \cap V$ is a disjoint union of two annuli.

Let $f_{n}: \mathbf{C P}^{1} \rightarrow \mathcal{N}(\Sigma)$ and $f_{\infty}: \mathbf{C P}^{1} \rightarrow \mathcal{N}(\Sigma)$ be the holomorphic maps associated to $\xi_{n}, \xi$ respectively (as in Section 5). Weak convergence of $\left[A_{n}\right]$ to $[A]$ is sufficient to ensure that the holomorphic map associated to $\Psi([A])$ is equal to $f_{\infty}$.

Note that gluing $\left.\left(E, \bar{\partial}_{A_{n}}\right)\right|_{\Sigma \times U}$ and $\left.\left(\operatorname{Id} \times f_{n}\right)^{*} \mathcal{U}\right|_{\Sigma \times V}$ along $W$ yields a bundle isomorphic to $\xi_{n}$ for all $n$. Also gluing $\left.\left(E, \bar{\partial}_{A}\right)\right|_{\Sigma \times U}$ and $\left.\left(\operatorname{Id} \times f_{\infty}\right)^{*} \mathcal{U}\right|_{\Sigma \times V}$ along $W$ yields a bundle in the equivalence class $\Psi([A])$.

Let $\tilde{E} \rightarrow S$ be the underlying smooth vector bundle of $\xi$ (and hence also of $\left.\xi_{n}\right)$, and let $X=\cup_{i=1}^{l} x_{i}$.

Now by Corollary 8.3, there exist $\bar{\partial}$-operators $\bar{\partial}_{n}$ on $\tilde{E}$ whose restriction to $U$ is equal to $\bar{\partial}_{A_{n}}$, and a $\bar{\partial}$-operator $\bar{\partial}_{\infty}$ on $\left.\tilde{E}\right|_{S-X}$ such that

- $\left(\tilde{E}, \bar{\partial}_{n}\right) \cong \xi_{n}$,

- after passing to a subsequence, $\bar{\partial}_{n} \rightarrow \bar{\partial}_{\infty}$ in $C^{\infty}$ on compact subsets of $S-X$, and

- $\left.\left(\left.\tilde{E}\right|_{S-X}, \bar{\partial}_{\infty}\right) \cong \Psi([A])\right|_{S-X}$.

Choose a metric $g$ on $S$ whose restriction to $U$ is equal to $g_{c y l}$, and a hermitian metric $H$ on $\tilde{E}$ whose restriction to $\left.\tilde{E}\right|_{\Sigma \times U}$ is pulled back from $E_{\Sigma} \rightarrow \Sigma$. Then we may form connections $A\left(H, \bar{\partial}_{n}\right)$ on $\tilde{E}$, where $A(H, \bar{\partial})$ denotes the unique connection which is compatible with the metric $H$ and the complex structure $\bar{\partial}$.

These connections satisfy the following properties:

- $A\left(H, \bar{\partial}_{n}\right)$ converge to $A\left(H, \bar{\partial}_{\infty}\right)$ in $C^{\infty}$ on compact subsets of $S-X$

- each $A\left(H, \bar{\partial}_{n}\right)$ is projectively ASD on $\Sigma \times U$ for all $n \gg 0$.

It follows from Lemma 8.5 that $\xi \cong \Psi([A])$. 


\section{From stable bundles to instantons}

We will show that the map $\Psi$ is surjective by proving the following result, where $e$ is some fixed element of $4 \pi^{2} \mathbf{Z}$ and $\mathcal{D}, c$ are given by (1),(2) in Section 2 .

Proposition 9.1 Let $\mathcal{E}$ be a rank 2 holomorphic bundle on $S$ with $[\mathcal{E}] \in$ $\mathcal{Z}_{(\mathcal{D}, c)}$. Then there exists a $U(2)$-instanton $A$ with $\Psi([A])=[\mathcal{E}]$.

It may be possible to prove this using Donaldson's evolution equation method directly, cf $[12,6]$; however the degeneracy of the Kähler metric $g_{c y l}$ on the 3 -manifolds $\Sigma \times S^{1} \times\{t\}$ may cause difficulties. We avoid this analysis by finding a sequence of instanton connections on $\mathcal{E} \rightarrow S$ which converges to a $U(2)$-instanton on $\Sigma \times S^{1} \times \mathbf{R}$. The proof uses two fundamental results: one is Donaldson's theorem, which says that any bundle on an algebraic surface which is stable with respect to a given Hodge metric admits a Hermitian-Einstein connection with respect to that Hodge metric. The other is Uhlenbeck's weak compactness for instantons.

Using these results it is quite straightforward to come up with a $U(2)$-instanton $A$ on $E \rightarrow \Sigma \times S^{1} \times \mathbf{R}$. What is perhaps surprising is that no energy is lost in the limiting process, and that $\Psi([A])=[\mathcal{E}]$.

Proof of Proposition 9.1. We begin by finding a sequence of connections on $\mathcal{E}$ which converge weakly giving a $U(2)$-instanton $A$.

Consider the sequence of Hodge metrics on $S=\Sigma \times \mathbf{C P}^{\mathbf{1}}$ given by $g_{n}=$ $g_{\Sigma}+g_{\mathbf{C P}^{1}, n}$, where

$$
\begin{gathered}
g_{\Sigma}=\text { chosen fixed Kähler metric on } \Sigma, \\
g_{\mathbf{C P}^{1}, 1}=\text { Fubini-Study metric on } \mathbf{C P}^{\mathbf{1}} .
\end{gathered}
$$

In local coordinates $\theta, t$ on $S^{1} \times \mathbf{R} \subset \mathbf{C P}^{1}$

$$
g_{\mathbf{C P}^{1}, 1}=\frac{1}{4 \pi \cosh ^{2} t}\left(d \theta^{2}+d t^{2}\right) .
$$

We may choose real numbers $0=T_{1}<T_{2}<\ldots$, with $T_{n} \rightarrow \infty$ as $n \rightarrow \infty$, and smooth Kähler metrics $g_{\mathbf{C P}^{1}, n}$ on $\mathbf{C P}^{\mathbf{1}}$ such that

$$
g_{\mathbf{C P}^{1}, n}= \begin{cases}d \theta^{2}+d t^{2} & \text { if } t \in\left[-T_{n}+1, T_{n}-1\right] \\ \frac{1}{4 \pi \cosh ^{2} t}\left(d \theta^{2}+d t^{2}\right) & \text { if } t \notin\left(-T_{n}, T_{n}\right)\end{cases}
$$

and the associated volume form $\omega_{\mathbf{C P}^{1}, n}$ satisfies

$$
\int_{\mathbf{C P}^{1}} \omega_{\mathbf{C P}^{1}, n}=n \text {. }
$$

Geometry 8 Topology, Volume 5 (2001) 
We will denote by $\omega_{n} \in \Omega^{1,1}(S, \mathbf{R})$ the Kähler form of the metric $g_{n}$. Then $\omega_{n}$ is a representative of the cohomology class $n \sigma+f$ in the Kähler cone $\mathcal{K}(S)$ (see Section 5).

The set $\mathcal{Z}_{(\mathcal{D}, c)}$ is contained in $\mathcal{M}_{(\mathcal{D}, c)}$, so that $\mathcal{E}$ is stable with respect to any element of the chamber $\mathcal{C}_{\sigma}$ in $\mathcal{K}(S)$. There is some $N \in \mathbf{N}$, which depends on $e$, such that $\left[\omega_{n}\right] \in \mathcal{C}_{\sigma}$ for all $n \geq N$.

Let $\tilde{E}$ denote the underlying smooth vector bundle of $\mathcal{E}$. Let $H_{n}$ be a hermitian metric on $\tilde{E}$ whose restriction to $\left.\tilde{E}\right|_{\Sigma \times\left[-T_{n}+1, T_{n}-1\right]}$ is pulled back from $E_{\Sigma} \rightarrow \Sigma$.

Then by a theorem of Donaldson $[4,7]$, for each $n \geq N$, there is a unique unitary connection $A_{n}$ on $\left(\tilde{E}, H_{n}\right)$, up to gauge, which determines a holomorphic structure isomorphic to $\mathcal{E}$ and is Hermitian-Einstein with respect to $g_{n}$. This means that with respect to $g_{n}, A_{n}$ is projectively anti-self-dual and $\operatorname{Tr} F_{A_{n}}$ is a harmonic representative for $-2 \pi i c_{1}(\mathcal{E})$.

Lemma 9.2 The sequence $\left\{\left[A_{n}\right]\right\}_{n \geq N}$ of connections on $\mathcal{E}$ described above has a subsequence which converges on compact subsets on the complement of a finite set of points in $\Sigma \times S^{1} \times \mathbf{R}$ to give a (finite energy) $U(2)$-instanton.

Proof The proof is a standard argument based on fundamental theorems of Uhlenbeck. See for example [15, Theorem 6.1.1].

It now follows from Lemma 8.8, with $\left[\xi_{n}\right]=[\xi]=[\mathcal{E}]$, that producing a $U(2)-$ instanton in this way from the holomorphic bundle $\mathcal{E}$ actually produces an inverse of the map $\Psi$, ie, that

$$
\Psi([A])=[\mathcal{E}]
$$

\section{$10 \Psi$ is a homeomorphism}

In this section we complete the proof of Theorem 1 by showing that the map

$$
\Psi: \mathcal{M}_{e} / \mathcal{I} \rightarrow \mathcal{Z}_{(\mathcal{D}, c)}
$$

is a homeomorphism. We have already seen that it is a bijection; we need to see that both $\Psi$ and its inverse are continuous.

The codomain $\mathcal{Z}_{(\mathcal{D}, c)}$ is first countable since it is a subspace of the Gieseker moduli space of $\mathcal{C}_{\sigma}$-stable torsion-free sheaves. The domain is also first countable since it is contained in the Hilbert manifold of finite-energy connections modulo gauge. Thus to prove continuity it suffices to show that $\Psi$ and $\Psi^{-1}$ preserve limits of sequences. 
Proposition $10.1 \Psi$ is continuous.

Proof Suppose $\left[A_{n}\right]$ converge (strongly) to $[A]$ in $\mathcal{M}_{e}$, for some $e \in 4 \pi^{2} \mathbf{Z}$. We must show that $\Psi\left(\left[A_{n}\right]\right) \rightarrow \Psi([A])$ in $\mathcal{M}_{(\mathcal{D}, c)}$.

Let $f_{n}: \mathbf{C P}^{\mathbf{1}} \rightarrow \mathcal{N}(\Sigma)$ and $f_{\infty}: \mathbf{C P}^{\mathbf{1}} \rightarrow \mathcal{N}(\Sigma)$ be the holomorphic maps associated to $\Psi\left(\left[A_{n}\right]\right), \Psi([A])$ respectively (see Section 5$)$. Let $\cup_{i=1}^{k} z_{i}$ be the marked points in $\mathbf{C P}^{1}$ such that $\Psi([A])$ has unstable restriction to $\Sigma \times\left\{z_{i}\right\}$. Let $\cup_{i=1}^{k_{n}} z_{i ; n}$ be the marked points for $\Psi\left(\left[A_{n}\right]\right)$.

Let $D$ be any open neighbourhood of $\cup_{i=1}^{k} z_{i}$ in $\mathbf{C P}^{\mathbf{1}}$. We will show that there exists some $N$ such that the restriction of $\Psi\left(\left[A_{n}\right]\right)$ to $\Sigma \times\{z\}$ is stable for all $z \notin D$ and for all $n \geq N$.

For any compact set $K \subset S^{1} \times \mathbf{R}$, it follows as in the proof of Proposition 8.4 that there exists $N_{K}$ such that none of the marked points $\cup_{i=1}^{k_{n}} z_{i ; n}$ are in $K-D$ for all $n \geq N_{K}$. Thus either there exists an $N$ as required above, or some subsequence $z_{i_{n} ; n}$ is converging to either 0 or $\infty$. But this would contradict the strong convergence $\left[A_{n}\right] \rightarrow[A]$ since it would imply that $\Psi([A])$ has a lower energy level than the limit of the sequence $\Psi\left(\left[A_{n}\right]\right)$.

It also now follows (as in the proof of Proposition 8.4) that $f_{n} \rightarrow f_{\infty}$ in $C^{\infty}$ on $\mathbf{C P}^{\mathbf{1}}-\cup_{i=1}^{k} z_{i}$.

By Proposition 8.4, it now follows that $\Psi\left(\left[A_{n}\right]\right) \rightarrow \Psi([A])$ in $\mathcal{M}_{(\mathcal{D}, c)}$ as required.

Proposition $10.2 \Psi^{-1}$ is continuous.

Proof Suppose $\left\{\left[\xi_{n}\right]\right\},[\xi]$ are elements of $\mathcal{Z}_{(\mathcal{D}, c)}$ with $\left[\xi_{n}\right] \rightarrow[\xi]$ as $n \rightarrow \infty$. By Propositions 7.1 and 9.1 there exist unique elements $\left[A_{n}\right] \in \mathcal{M}_{e} / \mathcal{I}$ with $\Psi\left(\left[A_{n}\right]\right)=\left[\xi_{n}\right]$. Uhlenbeck compactness implies that the sequence $\left[A_{n}\right]$ has a subsequence which is converging on compact sets on the complement of a finite set of points $x_{1}, \ldots, x_{l}$ to $[A]$, where $A$ is a $U(2)$-instanton with $e(A) \leq$ $e-4 \pi^{2} l$. By Lemma 8.8,

$$
[\xi]=\Psi([A]) .
$$

It follows that in fact $[A] \in \mathcal{M}_{e} / \mathcal{I}$. The same argument shows that any subsequence of $\left[A_{n}\right]$ has a subsequence converging to $[A]$; it follows that $\left[A_{n}\right]$ converge to $[A]$ as required. 
Remark 10.3 One could follow the argument in [13, Section 4] and show that if in fact bubbling occurs in the weak convergence $\left[A_{n}\right] \rightarrow[A]$, then the limit of the sequence $\Psi\left(\left[A_{n}\right]\right)$ will have sheaf singularities at the bubble points. This partially describes the extension of $\Psi$ (or rather $\Psi^{-1}$ ) to the compactifications of $\mathcal{M}_{e}$ and $\mathcal{Z}_{(\mathcal{D}, c)}$.

Remark 10.4 It is to be expected that the map $\Psi$ may carry more information. By considering some suitable weighted Sobolev completions it should be possible to compare the deformation complexes and Kuranishi models associated to $[A]$ and $\Psi([A])$, where $[A] \in \mathcal{M}_{e}$, following [10, Sections 4.3.3, 4.3.4], and hence show that $\Psi$ is an orientation-preserving isomorphism of real analytic ringed spaces.

\section{References}

[1] M Atiyah, R Bott The Yang-Mills equations over Riemann surfaces, Phil. Trans. R. Soc. Lond. A 308 (1982) 523-615

[2] P Braam, S Donaldson, Floer's work on instanton homology, knots and surgery, from: "The Floer Memorial Volume", Birkhäuser (1995) 195-281

[3] B Booß-Bavnbek, K Wojciechowski, Elliptic boundary problems for Dirac operators, Birkhäuser (1993)

[4] S Donaldson, Anti self-dual Yang-Mills connections over complex algebraic surfaces and stable vector bundles, Proc. London Math. Soc. 50 (1985) 1-26

[5] S Donaldson, Floer homology and algebraic geometry, from: "Vector Bundles in Algebraic Geometry: Durham 1993", Cambridge University Press (1995)

[6] S Donaldson, Boundary value problems for Yang-Mills fields, Journal of Geom. \& Physics 8 (1992) 89-122

[7] S Donaldson, P Kronheimer, The geometry of four-manifolds, Oxford University Press (1990)

[8] S Dostoglou, D Salamon, Instanton homology and symplectic fixed points, from: "Symplectic Geometry", London Math. Soc. Lecture Note Ser. 192, Cambridge Univ. Press (1993) 57-93

[9] R Friedman, Algebraic surfaces and holomorphic vector bundles, SpringerVerlag (1998)

[10] R Friedman, J Morgan, Smooth four-manifolds and complex surfaces, Springer-Verlag (1994)

[11] D Gilbarg, N Trudinger, Elliptic partial differential equations of second order, second edition, Springer-Verlag (1983) 
[12] G-Y Guo, Yang-Mills fields on cylindrical manifolds and holomorphic bundles I, II, Comm. Math. Phys. 179 (1996) 737-775, 777-788

[13] J Morgan, Comparison of the Donaldson polynomial invariants with their algebro-geometric analogues, Topology, 32 (1993) 449-488

[14] J Morgan, T Mrowka, On the gluing theorem for instantons on manifolds containing long cylinders, preprint

[15] J Morgan, T Mrowka, D Ruberman, The $L^{2}$ moduli space and a vanishing theorem for Donaldson polynomial invariants, International Press (1994)

[16] P Newstead, Introduction to moduli problems and orbit spaces, SpringerVerlag (1978)

[17] Z Qin, PhD Thesis, Columbia University (1990) 